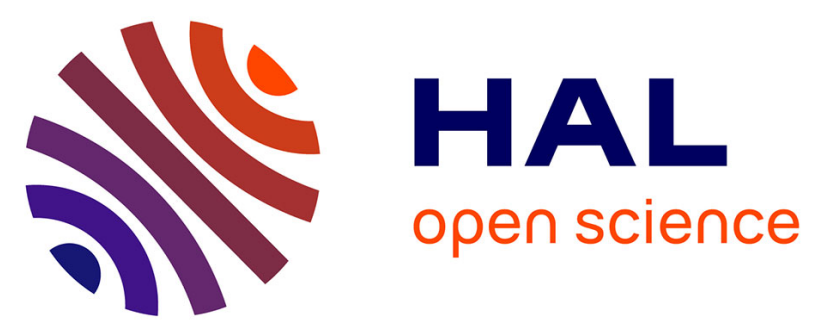

\title{
The effects of non-normality and nonlinearity of the Navier-Stokes operator on the dynamics of a large laminar separation bubble
}

\author{
Stefania Cherubini, Jean-Christophe Robinet, Pietro de Palma
}

\section{To cite this version:}

Stefania Cherubini, Jean-Christophe Robinet, Pietro de Palma. The effects of non-normality and nonlinearity of the Navier-Stokes operator on the dynamics of a large laminar separation bubble. The effects of non-normality and nonlinearity of the Navier-Stokes operator on the dynamics of a large laminar separation bubble, 2010, 22 (014102), pp.15. 10.1063/1.3276903 . hal-00799782

\section{HAL Id: hal-00799782}

\section{https://hal.science/hal-00799782}

Submitted on 12 Mar 2013

HAL is a multi-disciplinary open access archive for the deposit and dissemination of scientific research documents, whether they are published or not. The documents may come from teaching and research institutions in France or abroad, or from public or private research centers.
L'archive ouverte pluridisciplinaire HAL, est destinée au dépôt et à la diffusion de documents scientifiques de niveau recherche, publiés ou non, émanant des établissements d'enseignement et de recherche français ou étrangers, des laboratoires publics ou privés. 


\title{
The effects of non-normality and nonlinearity of the Navier-Stokes operator on the dynamics of a large laminar separation bubble
}

\author{
S. Cherubini, ${ }^{1,2}$ J.-Ch. Robinet, ${ }^{2}$ and P. De Palma ${ }^{1}$ \\ ${ }^{1}$ DIMeG, CEMeC, Politecnico di Bari, via Re David 200, 70125 Bari, Italy \\ ${ }^{2}$ SINUMEF Laboratory, Arts et Metiers ParisTech, 151 Bd. de l'Hôpital, 75013 Paris, France
}

(Received 8 October 2008; accepted 17 November 2009; published online 5 January 2010)

\begin{abstract}
The effects of non-normality and nonlinearity of the two-dimensional Navier-Stokes differential operator on the dynamics of a large laminar separation bubble over a flat plate have been studied in both subcritical and slightly supercritical conditions. The global eigenvalue analysis and direct numerical simulations have been employed in order to investigate the linear and nonlinear stability of the flow. The steady-state solutions of the Navier-Stokes equations at supercritical and slightly subcritical Reynolds numbers have been computed by means of a continuation procedure. Topological flow changes on the base flow have been found to occur close to transition, supporting the hypothesis of some authors that unsteadiness of separated flows could be due to structural changes within the bubble. The global eigenvalue analysis and numerical simulations initialized with small amplitude perturbations have shown that the non-normality of convective modes allows the bubble to act as a strong amplifier of small disturbances. For subcritical conditions, nonlinear effects have been found to induce saturation of such an amplification, originating a wave-packet cycle similar to the one established in supercritical conditions, but which is eventually damped. A transient amplification of finite amplitude perturbations has been observed even in the attached region due to the high sensitivity of the flow to external forcing, as assessed by a linear sensitivity analysis. For supercritical conditions, the non-normality of the modes has been found to generate low-frequency oscillations (flapping) at large times. The dependence of such frequencies on the Reynolds number has been investigated and a scaling law based on a physical interpretation of the phenomenon has been provided, which is able to explain the onset of a secondary flapping frequency close to transition. (C) 2010 American Institute of Physics. [doi:10.1063/1.3276903]
\end{abstract}

\section{INTRODUCTION}

In many engineering applications the boundary layer undergoes separation and reattachment, thus forming recirculation bubbles whose stability and control may be crucial for the performance of the device under consideration. This may happen, for example, over the surface of turbomachinery blades or of airplane wings. Separation may be triggered by the geometry of the body or by the adverse pressure gradient. In both cases the aerodynamic load may be strongly affected by the behavior of the bubble which changes its characteristics depending on the operating conditions. Often, the presence of a bubble is associated with a laminar-turbulent transition of the boundary layer since flow separation occurs in the laminar part of the bubble and, after transition, the flow reattaches. Such a transition is governed by the amplification of flow perturbations which may be due either to a linear process based on transient growth or to a nonlinear one in the presence of high free-stream disturbance levels (bypass transition). ${ }^{1,2}$ In Ref. 3, a thorough analysis is provided of the different transition mechanisms with respect to two- and three-dimensional initial perturbations, showing that several transition scenarios are possible and that, when small amplitude perturbations are considered, two-dimensional (2D) disturbances are the most amplified ones. For a separation induced by the wall geometry, such as a step ${ }^{4-7}$ or a bump, ${ }^{8-10}$ it has been established that the evolution of the perturbations leads to the formation of three-dimensional flow patterns (see also Ref. 11) characterized by global steady and weakly growing eigenmodes. Such three-dimensional steady modes have been originally discovered in Ref. 12 for a laminar separation bubble induced by an adverse pressure gradient. On the other hand, laminar separation bubbles show a strong 2D instability mechanism known as "flapping", $8,9,13,14$ and a high sensitivity to external noise ${ }^{15,16}$ whose basic features are still not fully understood. In particular, the following issues need to be investigated: (i) the role of the convective KelvinHelmholtz (KH) instability of the shear layer along the separation streamline with respect to the flapping phenomenon; (ii) the mechanism of transition from convective to global instability; and (iii) the influence of topological flow changes on the stability behavior (see Ref. 17 for a complete review).

The present work provides a stability analysis of the 2D flow over a flat plate with a separation bubble induced by a suction-and-blowing velocity profile. The aim of this paper is to describe the linear and nonlinear dynamics of a large separation bubble at low Reynolds numbers due to the nonorthogonality of the eigenvectors of the differential operator, leading to self-sustained oscillations and large transient amplifications of the initial disturbances. The linear dynamics, governed by the interactions among the nonorthogonal eigenvectors, is studied using a global eigenvalue analysis of the linearized Navier-Stokes (NS) equations. In fact, recently, several authors have demonstrated the suitability of 
the global eigenvalue analysis for studying the transient growth behavior by decomposing flow perturbations as a superposition of global modes. ${ }^{18}$ Such a technique has been applied to the study of several flows such as the boundarylayer flow over a flat plate, ${ }^{19-21}$ the separated boundary-layer flow induced by a bump, ${ }^{8-10}$ and the flow over a rounded backward-facing step. ${ }^{16}$ Indeed, the global analysis is capable of capturing the effects of the non-normality of the linearized NS operator and the consequent interactions among its nonorthogonal global modes. Nevertheless, it is still not clear which are the consequences of considering a finite number of modes. Therefore, a 2D direct numerical simulation (DNS) is employed as a complementary tool with respect to the global eigenvalue analysis for validating the results obtained by the eigenvalue analysis in the linear case and for studying the nonlinear dynamics of the separated flow. The numerical method is based on a fractional step approach for solving the NS equations, ${ }^{22}$ implementing a sixth-order-accurate discretization of the nonlinear terms based on a combined compact scheme for nonuniform grids. ${ }^{23}$ The global eigenvalue analysis is performed with respect to optimal perturbations of the base flow. Such optimal disturbances are computed by solving the singular value problem $^{1}$ associated with the differential operator and are superposed upon the base (steady) flow obtained by using the DNS. In order to compute the base flow for supercritical and slightly subcritical Reynolds numbers, a continuation strategy based on Newton's iteration is combined with the numerical method. ${ }^{24}$

Section II provides the definition of the problem, whereas in Sec. III the numerical tools are briefly described, namely, the DNS numerical algorithm, the continuation procedure based on Newton's iteration, and the global eigenvalue analysis. Then, a thorough discussion of the results is provided in Sec. IV for asymptotically stable and unstable flow regimes in cases of linear and nonlinear dynamics. Finally, some conclusions are drawn.

\section{GOVERNING EQUATIONS AND BOUNDARY CONDITIONS}

The 2D incompressible flow over a flat plate has been computed by solving the NS equations,

$$
\mathbf{u}_{t}+(\mathbf{u} \cdot \nabla) \mathbf{u}=-\nabla p+\frac{1}{\operatorname{Re}} \nabla^{2} \mathbf{u}
$$

$$
\nabla \cdot \mathbf{u}=0,
$$

where $\mathbf{u}=(u, v)^{T}$ is the velocity vector and $p$ is the pressure. Dimensionless variables are defined with respect to the inflow displacement thickness, $\delta^{*}$, and to the free stream velocity, $U_{\infty}$, so that the Reynolds number is equal to $\operatorname{Re}=U_{\infty} \delta^{*} / \nu$, where $\nu$ is the kinematic viscosity coefficient. A rectangular computational domain, with dimensions $L_{x}=420$ and $L_{y}=30$, is employed, $x$ and $y$ being the streamwise and wall-normal directions, respectively. At inlet points, placed at $x=65$ from the leading edge of the bottom wall, a Blasius boundary-layer profile is imposed for both the streamwise, $u$, and wall-normal, $v$, components of the veloc-

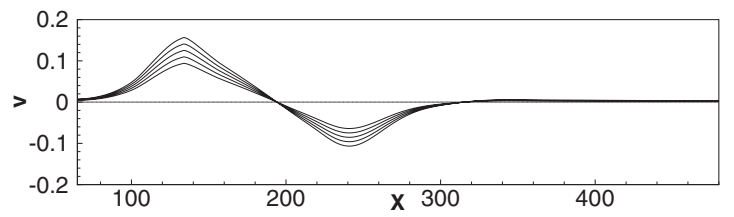

FIG. 1. Suction-and-blowing profiles imposed at the upper boundary for the $v$-component of the velocity.

ity vector, whereas at outlet points, a standard convective condition is employed. At the bottom wall, the no-slip boundary condition is prescribed. Finally, at the upperboundary points, a suction-and-blowing profile for the $v$-component of the velocity ${ }^{25}$ is imposed (five profiles with different magnitudes have been considered, see Fig. 1), and the vorticity is set to zero.

\section{NUMERICAL METHODS}

\section{A. 2D direct numerical simulation}

The NS equations are integrated by a fractional step method using a staggered grid. ${ }^{22}$ The viscous terms are discretized in time using an implicit Crank-Nicholson scheme, whereas an explicit third-order-accurate Runge-Kutta scheme is employed for the nonlinear terms. A second-orderaccurate centered space discretization has been used for the linear terms, whereas for the present calculations, a sixthorder-accurate space discretization has been implemented for the nonlinear terms based on a combined compact scheme for nonuniform grids. ${ }^{23}$ The basic idea of such a method is to relate the values of the unknown and of its first and second derivatives at three neighboring grid points. Moreover, since the NS equations are integrated on a staggered grid, a threepoint sixth-order-accurate interpolation formula has also been employed to compute the values of the unknowns at the cell centers.

All numerical simulations provided in the present work have been performed discretizing the computational domain by a $501 \times 150$ Cartesian grid stretched in the wall-normal direction, the height of the first cell close to the wall being equal to 0.1 . The Appendix provides a numerical gridconvergence study.

\section{B. Newton procedure for the base flow}

The above DNS method has been used to perform all the nonlinear simulations and to compute the base flow for the global stability analysis at subcritical Reynolds number. However, using the DNS, the residual cannot be reduced to machine zero when computing the base flow at supercritical as well as at slightly subcritical Reynolds numbers since some frequencies present in the numerical noise are amplified. In these cases, several approaches may be employed to compute the base flow based on filtering techniques ${ }^{26}$ or on continuation methods. Here, a time-stepping continuation method has been employed. Therefore, following the procedure proposed in Ref. 24, the DNS method has been combined with a Newton steady-state solver. The steady-state NS equations are written as 


$$
N(\mathbf{q})+L(\mathbf{q})=0,
$$

where $\mathbf{q}=(\mathbf{u}, p)$ and

$$
L(\mathbf{q})=\frac{1}{\operatorname{Re}} \nabla^{2} \mathbf{u}, \quad N(\mathbf{q})=-(\mathbf{u} \cdot \nabla) \mathbf{u}-\nabla p
$$

are the linear and nonlinear operators, respectively. In order to find the solution of Eq. (2), Newton's method is used. Starting from an initial solution $\mathbf{q}_{0}$, the variable $\mathbf{q}$ is iteratively updated by means of an increment $\delta \mathbf{q}$, computed by solving the following equation:

$$
\left(N_{q}+L\right)(\delta \mathbf{q})=(N+L)(\mathbf{q}),
$$

where $N_{q}$ is the linearized operator $N$. By choosing the operator $\Delta t(I-\Delta t L / 2)^{-1}$ as a preconditioner of Eq. (4), one obtains the following equation:

$$
\begin{aligned}
& {\left[\left(I-\frac{\Delta t L}{2}\right)^{-1}\left(I+\Delta t N_{q}+\frac{\Delta t L}{2}\right)-I\right](\delta \mathbf{q})} \\
& =\left[\left(I-\frac{\Delta t L}{2}\right)^{-1}\left(I+\Delta t N+\frac{\Delta t L}{2}\right)-I\right](\mathbf{q}) .
\end{aligned}
$$

It is noteworthy that the solution of Eq. (5) can be iteratively computed using the existing DNS algorithm with minor modifications since the fractional step operator, obtained by considering the implicit time discretization of the term $L(\mathbf{q})$, and the explicit discretization of $N(\mathbf{q})$,

$$
\mathbf{q}^{n+1}=\left(I-\frac{\Delta t L}{2}\right)^{-1}\left[I+\Delta t N+\frac{\Delta t L}{2}\right] \mathbf{q}^{n},
$$

have been formally recovered. The Newton method has been used for the computation of the base flows at supercritical Reynolds numbers and at slightly subcritical ones $(\operatorname{Re} \geq 207)$. In the subcritical case, the residual has been reduced to $10^{-12}$ in three up to ten Newton's iterations, whereas in the supercritical case, the iterations have been stopped when a residual level of $10^{-10}$ has been achieved due to a slower convergence of the algorithm.

\section{Global eigenvalue analysis}

Once the base flows have been computed for several values of the Reynolds number, their global stability is studied by means of a perturbative technique, namely, by considering the instantaneous variable $\mathbf{q}$ as a superposition of the base flow and of the perturbation $\mathbf{q}^{\prime}=\left(u^{\prime}, v^{\prime}, p^{\prime}\right)^{T}$. Such a perturbation is decomposed in temporal modes as

$$
\mathbf{q}^{\prime}(x, y, t)=\sum_{k=1}^{N_{t}} \kappa_{k}^{0} \hat{\mathbf{q}}_{k}(x, y) \exp \left(-i \omega_{k} t\right)
$$

where $N_{t}$ is the total number of modes, $\hat{\mathbf{q}}_{k}$ are the eigenvectors, $\omega_{k}$ are the eigenmodes (complex frequencies), and $\kappa_{k}^{0}$ represents the initial energy of each mode. A substitution of such a decomposition in Eq. (1) and a successive linearization lead to the following eigenvalue problem:

$$
\left(\mathbf{A}-i \omega_{k} \mathbf{B}\right) \hat{\mathbf{q}}_{k}=\mathbf{0}, \quad k=1, \ldots, N_{t} .
$$

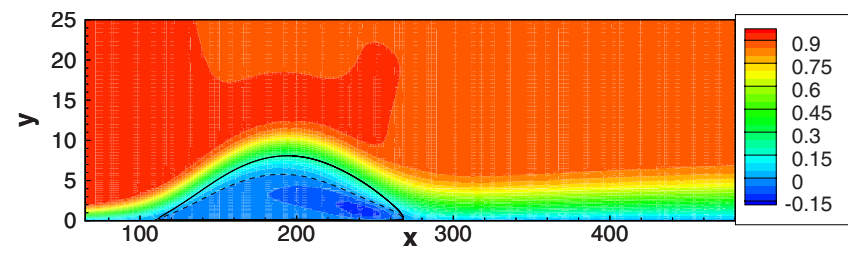

FIG. 2. (Color online) Streamwise velocity contours of the BF1 at Reynolds number $\operatorname{Re}=200$. The black line is the separation streamline, whereas the dashed line represents the $u=0$ contour.

The problem (8) is discretized with a Chebyshev/ Chebyshev collocation spectral method employing $N_{t}=850$ modes, and is solved with a shift-and-invert Arnoldi algorithm using the ARPACK library, ${ }^{27}$ the residual being reduced to $10^{-12}$. At the upper and inlet boundaries, a zero perturbation condition is imposed, whereas at the outflow, the flow being locally unstable, a Robin condition based on the approximation of the local dispersion relation is prescribed. ${ }^{20,21}$ Concerning the subcritical flow computations, the modes are discretized using $N_{x}=250$ collocation points in the $x$-direction and $N_{y}=48$ collocation points in the $y$-direction. The Appendix provides a numerical study for the grid sensitivity of the solution. For supercritical flow simulations, a slightly finer grid with $N_{x}=270$ and $N_{y}=50$ has been chosen due to the reduction in the boundary-layer displacement thickness.

\section{ANALYSIS OF THE RESULTS}

\section{A. Asymptotically stable dynamics: Transient growth and convective instabilities 1. Linear dynamics}

Figure 2 shows the streamwise velocity contours of the base flow 1 (BF1) at Reynolds number $\mathrm{Re}=200$, which has been obtained by imposing at the upper boundary the suction-and-blowing velocity profile with maximum magnitude shown in Fig. 1. BF2, BF3, BF4, and BF5 have been obtained by scaling such a profile by factors $0.9,0.8,0.7$, and 0.6 , respectively. A blowup of the separated zone is provided in Fig. 3 for all of the base flows, showing the decrease in the bubble size for decreasing values of the $v$ velocity imposed at the upper boundary. For such base flows, the global eigenvalue analysis has been performed. Figure 4 shows the spectra for $\mathrm{BF} 1, \mathrm{BF}$ 3, and $\mathrm{BF} 5$. All the spectra are found to be stable, although it can be noticed that for an increasing bubble size the eigenmodes rise up toward the $\omega_{i}=0$ axis. By inspecting the spectra, three families of modes can be detected, two of them having a very low growth rate. The asymptotic behavior of the flows is driven by the most un-

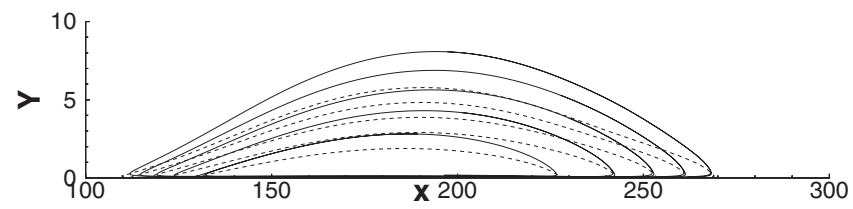

FIG. 3. Separation streamlines (solid lines) and streamwise zero-velocity contours (dashed lines) of BF1, BF2, BF3, BF4, and BF5 (from top to bottom) at Reynolds number $\mathrm{Re}=200$. 


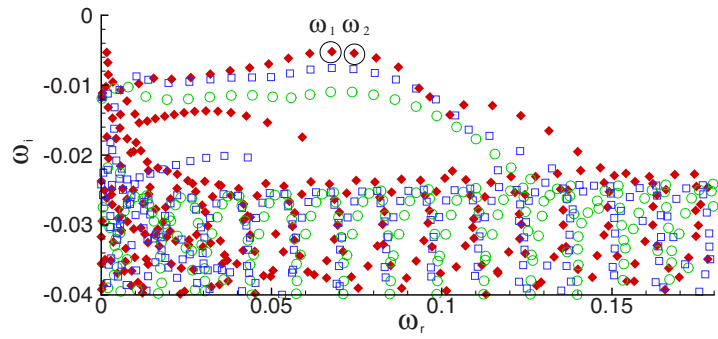

FIG. 4. (Color online) Eigenvalue spectrum for BF1 (diamonds), BF3 (squares), and BF5 (circles) at $\operatorname{Re}=200$. The modes labeled $\omega_{1}$ and $\omega_{2}$ are the most unstable ones.

stable modes, labeled $\omega_{1}$ and $\omega_{2}$ in Fig. 4 for BF1, located on the upper branch of the spectrum. Looking at the eigenvectors corresponding to such modes, shown in Figs. 5(a) and $5(\mathrm{~b})$, one can observe that they are reminiscent of the classical Tollmien-Schlichting (TS) modes predicted by a local approach. ${ }^{1}$ This is in agreement with previous results obtained by a global analysis for an attached laminar boundarylayer flow ${ }^{19-21}$ and for a cavity-induced separated boundarylayer flow. ${ }^{28}$ However, although the modes have been found asymptotically stable, they are likely to interact leading to a transient amplification of the perturbations due to the nonorthogonality of the corresponding eigenvectors. With the aim of measuring such an amplification, let us define the energy of the perturbations at time $t$ as

$$
E(t)=\frac{1}{2} \int_{0}^{L_{x}} \int_{0}^{L_{y}}\left(u^{\prime 2}+v^{\prime 2}\right) d x d y .
$$

Furthermore, the maximum energy gain $G(t)$, obtainable at time $t$ over all possible initial conditions, $\mathbf{u}_{0}^{\prime}$, is defined as

$$
G(t)=\max _{\mathbf{u}_{0}^{\prime} \neq 0} \frac{E(t)}{E(0)} .
$$

By decomposing the perturbation into the eigenmode basis (7), it is possible to rewrite Eq. (10) in the following form:
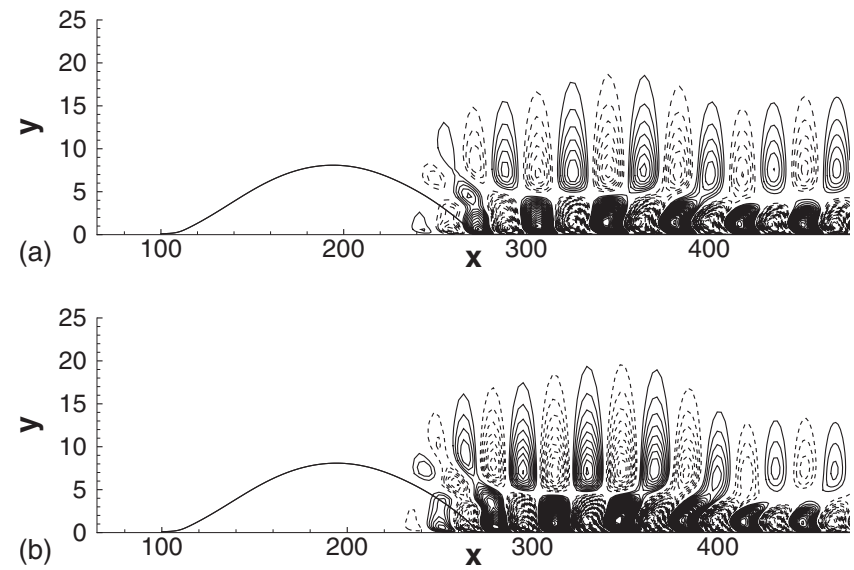

FIG. 5. Streamwise velocity components of the eigenvectors corresponding to the eigenvalues labeled (a) $\omega_{1}$ and (b) $\omega_{2}$ in Fig. 4. Solid-line contours indicate positive velocities; dashed-line ones are associated with negative velocities.

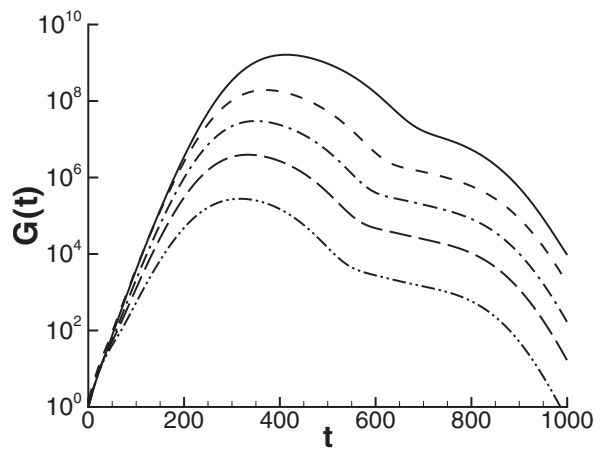

FIG. 6. Optimal energy gain curves at $\mathrm{Re}=200$ computed by the global eigenvalue analysis for BF1 (solid line), BF2 (dashed line), BF3 (dasheddotted line), BF4 (long-dashed line), and BF5 (dashed-dotted-dotted line).

$$
G(t)=\left\|\mathbf{F} \exp (-i t \boldsymbol{\Lambda}) \mathbf{F}^{-1}\right\|_{2}^{2},
$$

where $\boldsymbol{\Lambda}$ is the diagonal matrix of the eigenvalues, $\omega_{k}$, and $\mathbf{F}$ is the Cholesky factor of the energy matrix, $\mathbf{M}$, of components,

$$
M_{i j}=\iint\left(\hat{u}_{i}^{*} \hat{u}_{j}+\hat{v}_{i}^{*} \hat{v}_{j}\right) d x d y, \quad i, j=1, \ldots, N
$$

where the superscript $*$ denotes the complex conjugate. Finally, the maximum amplification at time $t$ and the corresponding optimal initial condition, $\mathbf{u}_{0}^{\prime}$, are computed by a singular value decomposition of the matrix $\mathbf{F} \exp (-i t \boldsymbol{\Lambda}) \mathbf{F}^{-1}$.

Figure 6 provides the maximum energy gain $G(t)$ obtained for BF1, BF2, BF3, BF4, and BF5 by choosing $N=600$ modes among the $N_{t}$ total modes employed for the global eigenvalue analysis. A numerical study of the sensitivity of the optimal energy gain curve with respect to the number of modes $N$ is provided in the Appendix. The energy gain curves for the five base flows here considered show a similar shape, although the maximum value of the energy gain, $G_{\max }$, as well as the time at which such a value is achieved, increases with the bubble size. Figure 7 provides the variation in $G_{\max }$ with respect to three features of the base flows, namely, the maximum value of the suction velocity at the upper boundary, $v_{\max }$; the maximum value of the shape factor, $H=\delta^{*} / \Theta$ (where $\Theta$ is the momentum thickness of the boundary layer); and the aspect ratio of the bubble, which is defined as the ratio between the maximum height of the separated region, $h$ (measured at the zero-streamwisevelocity line), and the length of the bubble. Figure 7 shows an approximatively linear increase in the $G_{\max }$ with respect to all of the three parameters. It is noteworthy that the aspect ratio of the smallest bubble is close to the ones experimentally measured for laminar separation bubbles on the suction surface of aerofoils at a large angle of attack, ${ }^{29,30}$ whereas the largest bubble has a shape factor which is comparable to the one analyzed in Refs. 3 and 31.

The dashed line in Fig. 8 shows the linear transient time evolution of the energy gain, $E(t) / E(0)$, obtained by the global eigenvalue analysis using the initial perturbation, $\mathbf{u}_{0}^{\prime \max }$, which provides the maximum peak value at $\mathrm{Re}=200$ for 

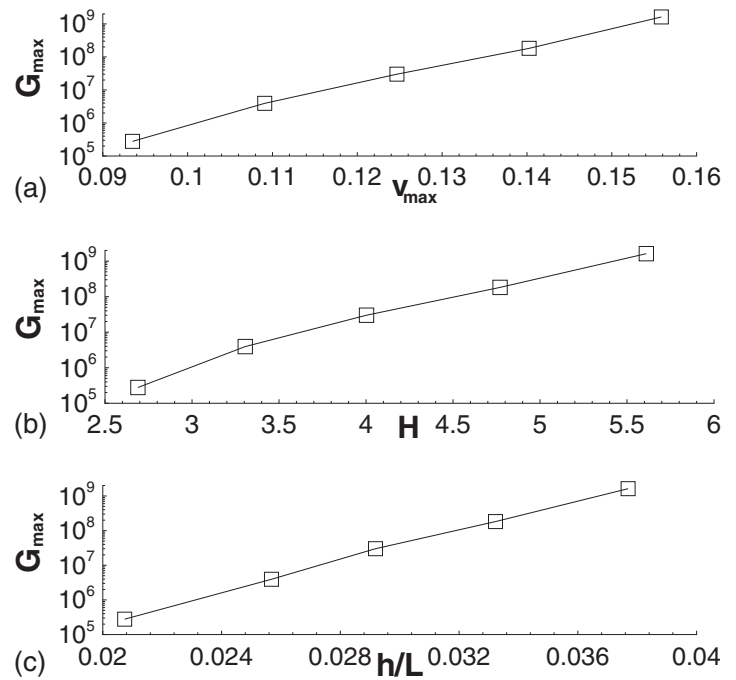

FIG. 7. Maximum value of the optimal energy gain computed by the global eigenvalue analysis at $\mathrm{Re}=200$ vs (a) the maximum suction velocity at the upper boundary, (b) the shape factor, and (c) the aspect ratio for BF1, BF2, $\mathrm{BF} 3, \mathrm{BF} 4$, and $\mathrm{BF} 5$ (from right to left).

BF1. Such a curve is almost coincident with the $G(t)$ one, shown by the solid line in Fig. 8 for the same number of modes, $N=600$. Both curves reach a maximum value of order of magnitude $10^{9}$ at $t=410$, meaning that the linearized operator related to the considered flow has a high degree of non-normality.

In order to get some insight into the amplification mechanism, the evolution of such an optimal perturbation in time is analyzed. Figure 9(a) shows that at time $t=0$ the energy of the optimal perturbation is concentrated in the upstream part of the bubble. At $t=200$ [Fig. 9(b)], the disturbance has been convected downstream by the base flow along the separation streamline through a $\mathrm{KH}$ mechanism, and it has been amplified before reaching the reattachment point. Such an amplification is due to the local convective instability of the velocity profiles within the bubble, which leads to a global growth of the perturbations, as theoretically demonstrated in Ref. 32 using the Ginzburg-Landau equation for nonparallel flows. After the reattachment point [Fig. $9(\mathrm{c})]$, the perturbation is convected through the attached

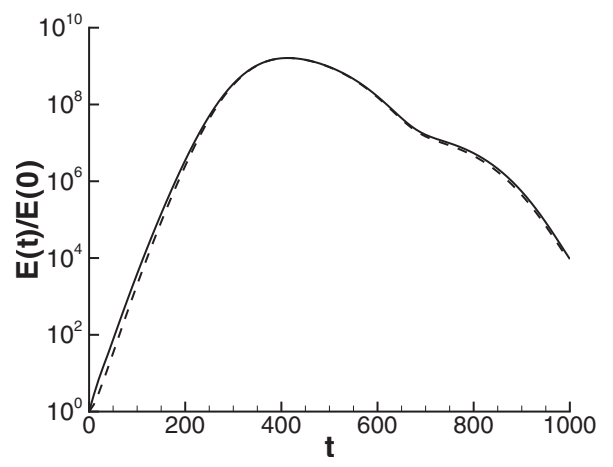

FIG. 8. Optimal energy gain curve (solid line) and evolution of the normalized energy corresponding to the initial perturbation giving the optimal energy peak (dashed line) at $\mathrm{Re}=200$, both computed by the global eigenvalue analysis for BF1.
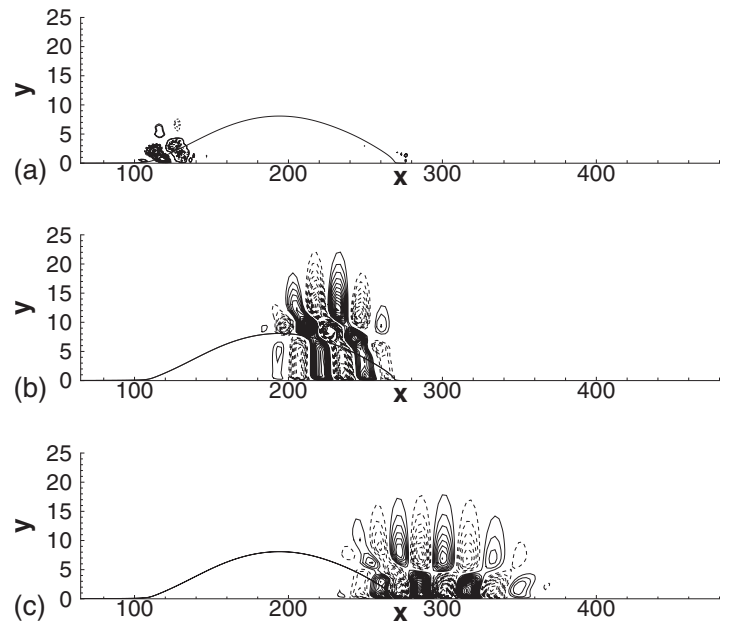

FIG. 9. Streamwise velocity contours of the optimal perturbation obtained by the global eigenvalue analysis for BF1 at time (a) $t=0$, (b) $t=200$, and (c) $t=400$. Solid-line contours indicate positive velocities; dashed-line ones are associated with negative velocities.

boundary layer, where it is damped. The same convective mechanism has been recovered for BF2, BF3, BF4, and BF5, explaining the linear increase in $G_{\max }$ and of the time at which it is reached for an increase in the bubble size. It is worth to notice that the energy gain values recovered by the global eigenvalue analysis are quite high. Nevertheless, one has to consider that these are optimal values, therefore the amplification for a real perturbation could be much lower. It is anticipated that for small bubbles, the amplification of the disturbances could be too low to induce nonlinear effects. For this reason, an investigation will be performed of the amplification of a random white-noise disturbance, forced at the inlet or in the whole domain.

Due to the similarities recovered in the transient behavior of the different base flows, the following analysis will be carried out only for the separated flow BF1.

\section{Weakly nonlinear dynamics}

In order to validate the results of the linear stability analysis and to study the weakly nonlinear behavior of the considered separated flow, the DNS has been performed initializing the simulation by superposing the optimal perturbation upon the base flow. In order to satisfy the hypothesis of small perturbations, thus allowing a comparison with the results of the global eigenvalue analysis, the optimal disturbance, $\mathbf{u}_{0}^{\prime \max }$, has been scaled by a factor $A_{0}=10^{-8}$, which is four orders of magnitude greater than the residual noise. The energy of the disturbance defined in Eq. (9), normalized by the value at $t=0$, has been computed at each time step and is reported in Fig. 10 using the dashed line. In order to verify that a perturbation of order of magnitude $A_{0}=10^{-8}$ is small enough to allow a meaningful comparison with the linear model, a linearized DNS has been performed as well, whose result, shown by the dotted line in Fig. 10, has been found identical to the one obtained by the DNS. Indeed, by injecting an initial perturbation with order of magnitude $10^{-8}$, which corresponds to an initial energy of order of magnitude $10^{-16}$, such a perturbation amplifies itself up to a factor of 


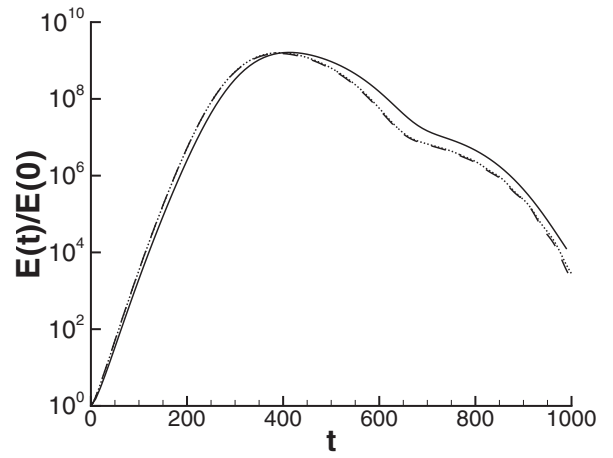

FIG. 10. Time evolution of the energy gain of the optimal initial perturbation obtained by the global eigenvalue analysis (solid line), by the DNS (dashed line), and by the linearized DNS (dotted line).

$10^{9}$, reaching an energy level about equal to $10^{-7}$, which is low enough for nonlinear effects to be negligible. Moreover, Fig. 10 shows that the optimal perturbation energy growth curve obtained with the global eigenvalue analysis, provided by the solid line, is very close to the one computed by the DNS (dashed line), validating the capability of the global model to predict the linear transient mechanism.

However, although the DNS has confirmed the convective amplifier character of the considered separated flow for an optimal initial perturbation, there is no evidence that such a flow would behave similarly in a real case. For this reason, simulations have been performed in which the base flow is perturbed using a time-varying pseudorandom zero-mean Gaussian white-noise disturbance. The flow has been perturbed in two different ways. In the first case (A), a disturbance field is impulsively injected in the whole domain. In the second case (B), the perturbation is superposed upon the inlet velocity profile, as it may happen in real experiments, where the inlet flow may be affected by some noise. In both cases a strong energy gain has been observed, which is approximatively two orders of magnitude lower than the optimal one. As shown in Fig. 11, the shape of the amplification curves is not far from the optimal one, although some differences can be noticed. In particular, the algebraic growth phase is delayed with respect to the optimal case. This is due

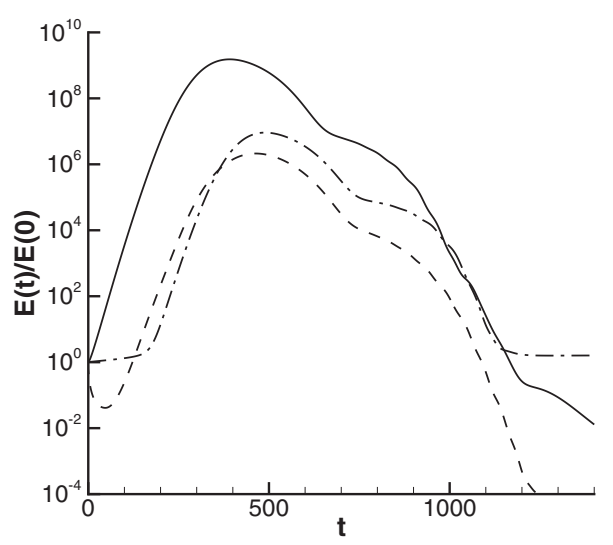

FIG. 11. Time evolution of the energy gain computed by the DNS for an optimal initial perturbation (solid line), for a disturbance field injected in the whole domain (case A, dashed line), and for a time-varying disturbance superposed upon the inlet velocity profile (case B, dashed-dotted line).

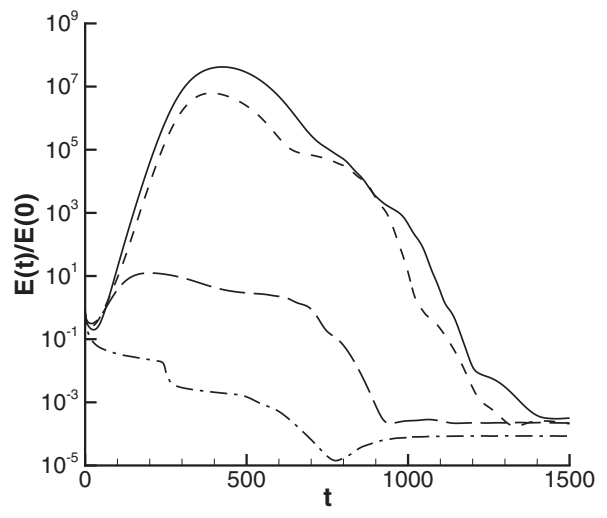

FIG. 12. Time evolution of the energy gain computed by the DNS for an initial perturbation placed upstream (solid line), downstream (dashed-dotted line), or within the bubble in its first half (short-dashed line) or in its second half (long-dashed line).

to the fact that the perturbation is damped until it is convected by the base flow along the separation streamline, where it begins to be amplified. Due to such an initial delay, the time instant at which the amplification peak occurs $\left(t_{\max _{\mathrm{A}}}=460\right.$, for case $\mathrm{A}$, and at $t_{\text {max }_{\mathrm{B}}}=490$, for case B) is greater with respect to the optimal case. It is noteworthy that for case $\mathrm{B}$, in which the inflow perturbation is continuously injected into the flow, the normalized energy does not decay for $t<150$ and $t>1100$, but assumes a value slightly greater than 1. Indeed, for $t>1100$, after the first wave packet has been advected through the separated zone, a statistically steady state is established, so that the continuously injected perturbations do not experience more transient amplification. Nevertheless, some highly sensitive frequencies are excited by the random forcing at the inlet, ${ }^{33}$ and are slightly amplified also after the transient has passed, so that the energy gain maintains asymptotically a value close to 1 . Therefore, it is possible to conclude that even though the base flow is continuously perturbed, its response to a small amplitude perturbation is comparable to the response to an impulsive perturbation, which could mean that the strong transient amplification of the perturbations is a robust feature of separated flows. Such results are in agreement with previous ones obtained for a flow over a backward facing step perturbed by an inflow random disturbance. ${ }^{6,33}$

Finally, in order to understand the role of the separated region on such a dynamics, several DNSs have been performed in which the perturbation is respectively placed upstream $(70<x<100)$, downstream $(280<x<340)$, and within the bubble in its first half $(120<x<180)$ or in its second half $(200<x<260)$. Figure 12 shows that a perturbation placed upstream or within the bubble is amplified, whereas a disturbance initially located downstream of the bubble is damped. In particular, the dynamics of a perturbation placed in the first half of the bubble is comparable to the dynamics of case A, whereas a perturbation placed in the second half of the bubble is only weakly amplified, confirming that the amplification mechanism is based on the convection of perturbations along the separation streamline. 


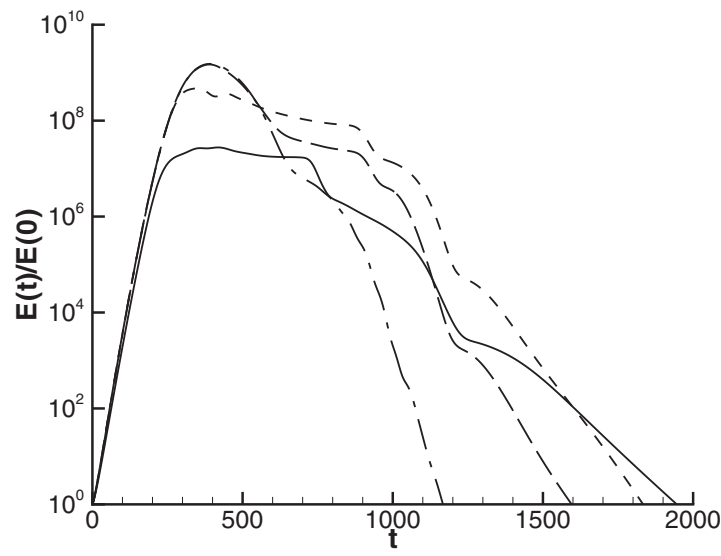

FIG. 13. Time evolution of the energy gain computed by the DNS for an optimal initial perturbation with order of magnitude $A_{0}=10^{-4}$ (solid line), $A_{0}=10^{-5}$ (short-dashed line), $A_{0}=10^{-6}$ (long-dashed line), and $A_{0}=10^{-8}$ (dashed-dotted line).

\section{Nonlinear dynamics and sensitivity response}

In order to study the role of nonlinear effects in the dynamics of a separated flow, nonlinear simulations have been performed increasing the amplitude of the initial perturbation, $\mathbf{u}_{0}^{\prime \max }$. Figure 13 provides the energy gain curves obtained scaling the optimal perturbation by a factor $A_{0}$ equal to $10^{-8}, 10^{-6}, 10^{-5}$, and $10^{-4}$, respectively. All of the curves initially follow the algebraic growth phase, but, for amplitudes greater than $10^{-6}$ they show a reduced peak value with respect to the linear case. For $A_{0}=10^{-4}$, a saturation plateau starts immediately after the initial algebraic growth phase around a value about equal to $10^{7}$. For all cases, the perturbations eventually decay. It is worth to notice that for
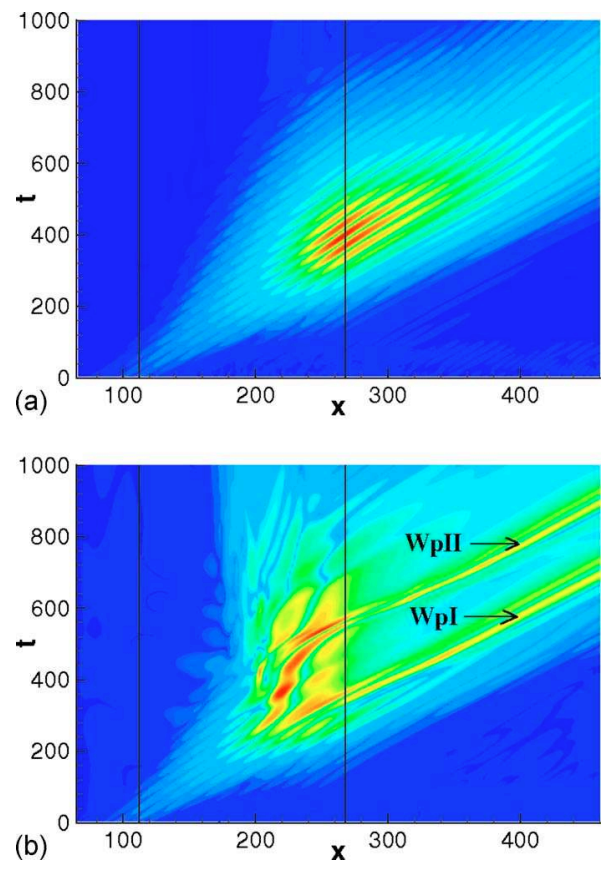

FIG. 14. (Color online) Space-time diagram of the vorticity perturbation at wall, $\omega_{z}^{\prime}$, computed by DNS for an optimal initial perturbation normalized by factors (a) $A_{0}=10^{-8}$ and (b) $A_{0}=10^{-4}$. The black lines indicate the separation and reattachment points of the base flow, whereas the arrows point at the two wave packets shed by the bubble (WpI and WpII).

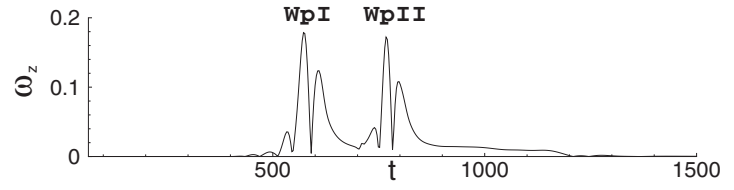

FIG. 15. Time evolution of the vorticity perturbation at wall computed by DNS for an optimal initial perturbation with order of magnitude $A_{0}=10^{-4}$ at the streamwise location $x=400$.

$A_{0} \geq 10^{-6}$, the decaying rate is lower than the linear one due to the capability of the nonlinear terms to transfer the energy back in the upstream part of the bubble. It is possible to visualize such a mechanism by inspecting, at a fixed wall normal position, the evolution in time of the perturbation that propagates along the streamwise direction. Indeed, comparing the evolution of the linear and nonlinear wave packets shown in Fig. 14, one can see that in the second case [Fig. 14(b)], a part of the perturbation is driven back in the separated region, where it interacts with the main part of the wave packet $(\mathrm{WpI})$ which is being convected downstream. As a result, a second wave packet (WpII) appears in the bubble, which is convected in the attached-flow region. Looking at Fig. 15, which shows the evolution in time of the vorticity perturbation, $\omega_{z}^{\prime}$, at wall at a fixed streamwise position $(x=400)$, it is possible to notice that the amplitude of the two wave packets convected downstream is comparable, meaning that a wave packet cycle starts to be established within the bubble, but it is eventually damped.

In order to study the development of the wave packet downstream of the bubble, a longer computational domain has been considered, $L_{x}^{\prime}=5 L_{x}$. As shown in Fig. 16, for $A_{0}=10^{-4}$ and $1000<t<4000$, the perturbation is amplified, whereas for $A_{0}=10^{-8}$ and $t>1000$, it is damped when the same domain length is used. Looking at the evolution of the wave packet in time, provided in Fig. 17, it is possible to notice that the perturbation is amplified while it is convected

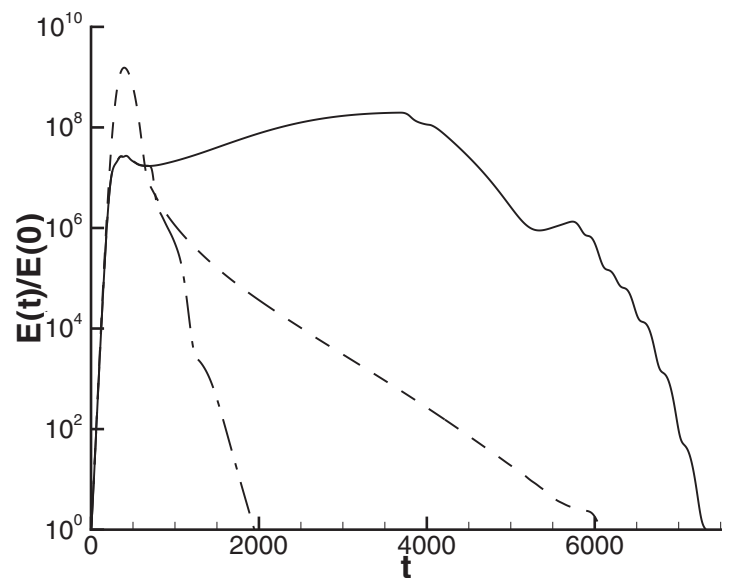

FIG. 16. Time evolution of the energy gain computed by the DNS for an initial optimal perturbation with order of magnitude $A_{0}=10^{-4}$ with domain lengths $L=L_{x}$ (dashed-dotted line) and $L=5 L_{x}$ (solid line), and for an initial optimal perturbation with order of magnitude $A_{0}=10^{-8}$ and domain length $L=5 L_{x}$ (dashed line). 
(a)

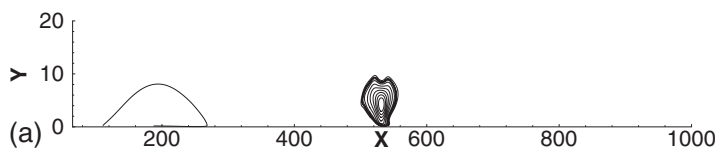

(b)

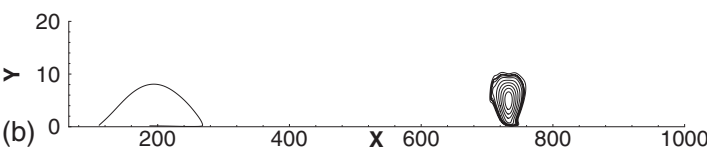

(c)

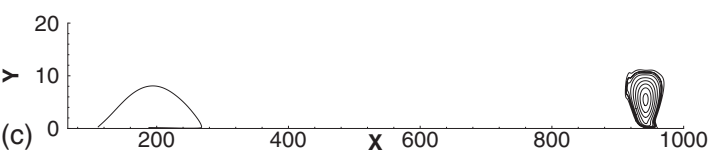

FIG. 17. Contours of the streamwise velocity perturbation, $u^{\prime}$, computed by the DNS at three time instants: (a) $t=2200$, (b) $t=2600$, and (c) $t=3000$. The line on the left indicates the separation streamline.

through the attached-flow region, causing a further transient global growth of the energy until it leaves the domain. Such a result indicates that the flow downstream of the bubble is convectively nonlinearly unstable although linearly globally stable. In order to understand the mechanism inducing such an amplification, the Fourier transform in time of the streamwise-velocity fluctuation at two different abscissas, $x$ $=380$ and $x=455$, has been computed. Figure 18 shows that immediately downstream of the bubble, the leading mode corresponds to the less stable eigenvalue of the global spectrum, labeled $\omega_{1}$ in Fig. 4, whose real part is $\omega_{r} \approx 0.08$. Thus, at such a location the behavior of the flow is driven by the global dynamics. However, for increasing abscissas, the dynamics is driven by a different mode whose real part is close to $\omega_{r} \approx 0.045$. A local spatial stability analysis, which solves the Orr-Sommerfeld and Squire eigenvalue problem with respect to a streamwise wave number $\alpha$, has been performed on the velocity base flow profiles at the above streamwise locations. This analysis indicates that such a mode lies in the range of the local spatially unstable frequencies for $x=455$, as shown in Fig. 19. The same analysis has been performed at several streamwise locations downstream of the bubble confirming such a result. Thus, one can infer that the wave packet is spatially amplified while it is convected downstream as a consequence of the excitation of a locally unstable mode. In order to find the "critical perturbation" triggering such a mode, several simulations have been performed with increasing initial perturbation amplitudes. The streamwise velocity perturbations have been extracted as

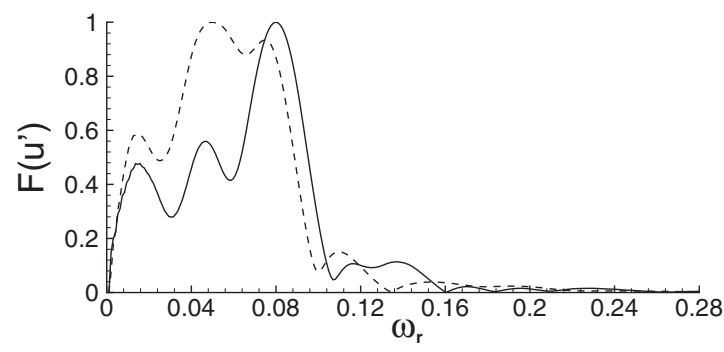

FIG. 18. Fourier spectrum in time of the streamwise velocity perturbation at the first grid point in the wall normal direction computed by the DNS for two streamwise locations: $x=380$ (solid line) and $x=455$ (dashed line).

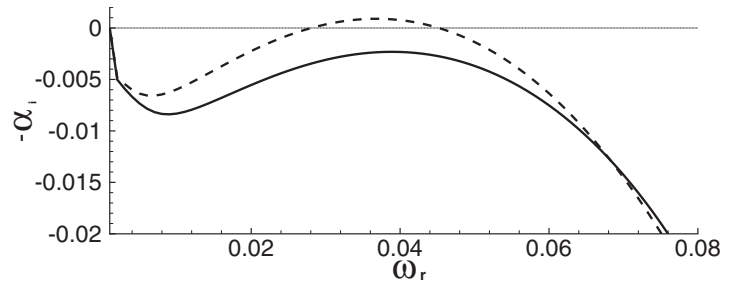

FIG. 19. Spatial amplification rate, $-\alpha_{i}$, vs the real pulsation, $\omega_{r}$, computed by local eigenvalue analysis at two streamwise locations: $x=380$ (solid line) and $x=455$ (dashed line).

they have passed beyond the location $x=455$ at two time instants, $t=900$ and $t=1000$. As shown in Fig. 20, a spatial amplification begins to be noticed for amplitudes equal to $10^{-6}$. Such a strong influence of the perturbation amplitude on the behavior of the flow could be due to a high sensitivity to real forcing. Indeed, although the flow is not directly forced with a specific mode, several frequencies are present in the perturbed flow due to the initial impulsive forcing, which could eventually be damped or not depending on the sensitivity of the flow.

The sensitivity of the flow has been studied by adding a forcing term, $\hat{\mathbf{q}}_{f} e^{-i \sigma t}$, to the linear evolution equation (8), $\sigma$ being a real frequency. ${ }^{34}$ The solution of the problem is

$$
\hat{\mathbf{q}}=\hat{\mathbf{q}}_{0} e^{\mathbf{D} t}-\hat{\mathbf{q}}_{f} e^{-i \sigma t} /(i \sigma \mathbf{B}-\mathbf{A}),
$$

where $\hat{\mathbf{q}}_{0}$ is the initial condition and $\mathbf{D}$ is the diagonal matrix, $D_{k, l}=-i \delta_{k, l} \omega_{k}$. Since the flow is globally stable, the solution for long times is governed by the term $-\hat{\mathbf{q}}_{f} e^{-i \sigma t} /(i \sigma \mathbf{B}-\mathbf{A})$. Moreover, since the influence of an external real harmonic forcing is determined for long times by $\hat{\mathbf{q}}_{f}$, it is possible to compute the sensitivity to a real external frequency through the analysis of the norm $\left\|(i \sigma \mathbf{B}-\mathbf{A})^{-1}\right\| .^{34}$ Such an analysis is performed through the evaluation of the pseudospectrum of the global linear operator defined as

$$
\lambda_{\varepsilon}=\left\{\sigma \in \mathbb{C},\left\|(i \sigma \mathbf{B}-\mathbf{A})^{-1}\right\| \geq \varepsilon^{-1}\right\} .
$$

Figure 21 provides the pseudospectrum of the flow considered here, represented plotting the contours of $-\log _{10}(\varepsilon)$. At each point on the real axis $\left(\omega_{i}=0\right)$, the contour value represents the sensitivity of the flow to external forcing with the corresponding pulsation $\omega_{r}$. The response to a real frequency
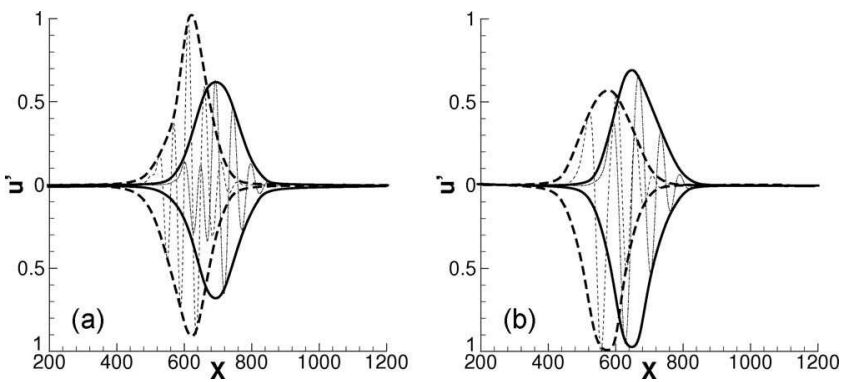

FIG. 20. Evolution in the streamwise direction of the streamwise perturbation velocity, $u^{\prime}$, computed by the DNS at a fixed wall normal position, $y=1.49$, for two time values, $t=900$ (dashed line) and $t=1000$ (solid line), for initial perturbations scaled by factors (a) $A_{0}=10^{-7}$ and (b) $A_{0}=10^{-6}$, respectively. 


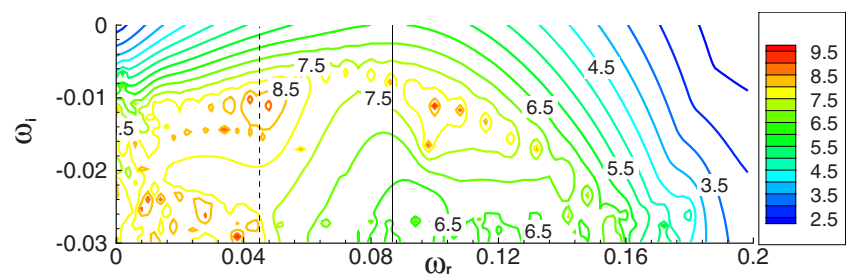

FIG. 21. (Color online) Pseudospectrum contours represented using the logarithmic scale $-\log _{10}(\varepsilon)$. The vertical solid line indicates the most sensitive pulsation $\left(\omega_{r} \approx 0.085\right)$, whereas the vertical dashed line indicates the sensitivity value to a forcing of pulsation $\omega_{r} \approx 0.045$.

is dominated by the global KH/TS-like modes, the most sensitive one corresponding to the most unstable one. ${ }^{15}$ Concerning the locally unstable mode with $\omega_{r} \approx 0.045$, it lies in the range of the highly sensitive modes. As shown in Fig. 21, the minimal perturbation amplitude triggering the real frequencies around $\omega_{r} \approx 0.045$ is approximatively $10^{-6}$, which matches the value previously found by DNS (see Fig. 20).

Finally, in order to investigate if such a mechanism linking sensitivity and convective instability may be found also in other flow configurations, several DNSs with increasing Reynolds number have been performed. At $\mathrm{Re}=223$, an asymptotically unstable dynamics is recovered by superposing upon the base flow a perturbation of order of magnitude $A_{0}=10^{-4}$, while asymptotic stability is obtained with a smaller perturbation. As shown in Fig. 22, the normalized energy saturates at a value of $10^{9}$, which is maintained asymptotically, leading to a periodic self-sustained state. Since the period of the oscillations is about $T \approx 110$, it is possible to identify the locally unstable pulsation, $\omega \approx 0.055$, which is very close to the one previously found for $\mathrm{Re}=200$. Therefore, it is likely that the generation of such a self-sustained state is due to the high sensitivity of the flow to that locally unstable frequency which leads the flow to a subcritical transition. Such results show that the convective modes are a relevant feature of a separated boundary layer flow, being able to arise from different mechanisms (such as nonnormality and sensitivity) in both a linear and a nonlinear framework, and are able to play an active role in subcritical transition.

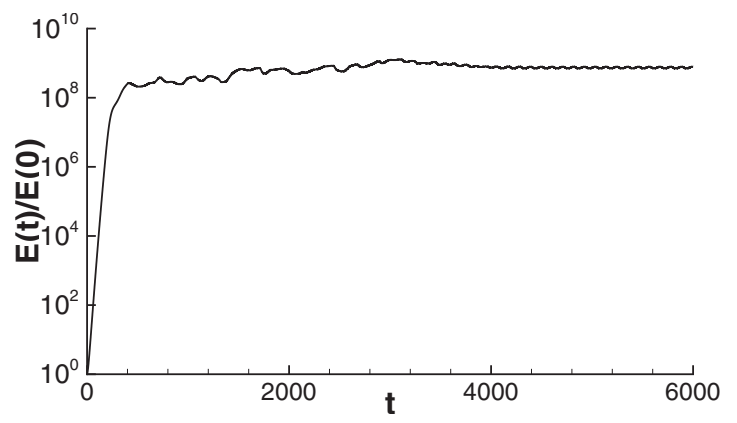

FIG. 22. Time evolution of the energy gain computed by the DNS for an initial optimal perturbation of order of magnitude $A_{0}=10^{-4}$ at $\operatorname{Re}=223$.

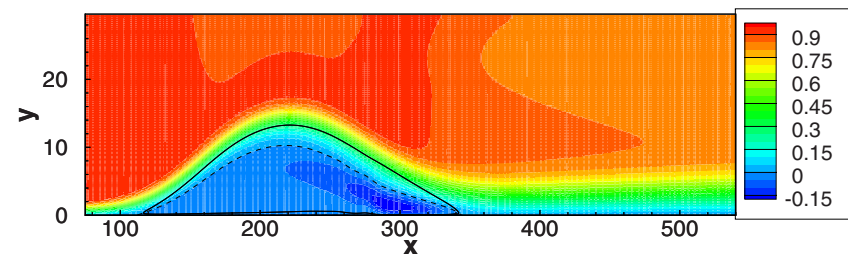

FIG. 23. (Color online) Streamwise velocity contours of the base flow at Reynolds number $\operatorname{Re}=225$. The solid line is the separation streamline, whereas the dashed line represents the $u=0$ contour.

\section{B. Asymptotically unstable dynamics: The origin of unsteadiness and the flapping frequency}

\section{Linear dynamics}

The supercritical dynamics of the flow has been investigated by performing the global eigenvalue analysis with increasing Reynolds numbers. Transition has been found to occur at $\mathrm{Re}=225$; Fig. 23 shows the corresponding streamwise velocity contours of the base flow. The structure of the spectrum at such a Reynolds number, provided in Fig. 24, is quite similar to the one at $\mathrm{Re}=200$. The spectrum is marginally unstable, since there are seven slightly unstable modes placed on the convective branch, their eigenvectors being reminiscent of the TS waves. Comparing Fig. 23 with Fig. 2, it is possible to notice that for increasing Reynolds numbers, not only the bubble size (defined as the length of the separation region at the wall) increases, but some topological changes occur in the base flow. Looking at the separation streamline, one can observe a smoother reattachment and, most importantly, the presence of a secondary separation zone within the primary one, which is identified by a change in sign of the skin friction coefficient within the primary bubble. Such observations support the hypothesis ${ }^{12,35}$ that topological changes in the base flow could be at the origin of the onset of the unsteadiness in separation bubbles. As suggested in Ref. 10, it is likely that the primary instability of the considered bubble would be of structural type, meaning that for a supercritical Reynolds number the flow cannot exhibit any single-bubble state, instead it is characterized by a multiple separation which induces the vortex formation and shedding, leading to asymptotical instability.

The linear energy growth has been studied using the global eigenvalue analysis for increasing Reynolds numbers. The behavior of the corresponding energy-gain curves, reported in Fig. 25, shows that (i) the first peak value increases linearly with respect to $\mathrm{Re}$, and (ii) the time at which the first

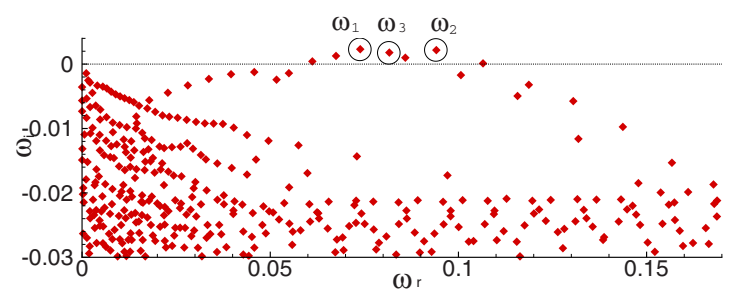

FIG. 24. (Color online) Eigenvalue spectrum for the flow at $\mathrm{Re}=225$ with $N_{x}=270$ and $N_{y}=50$ grid points. The modes labeled $\omega_{1}, \omega_{2}$, and $\omega_{3}$ are the most unstable ones. 


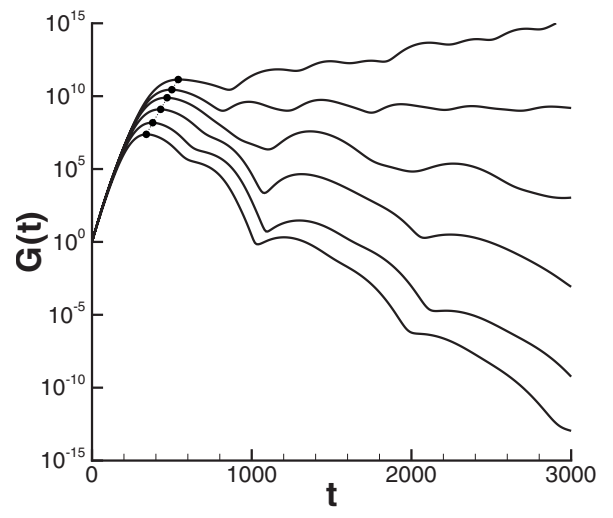

FIG. 25. Optimal energy gain curves obtained by the global eigenvalue analysis with $N=400$ modes for increasing Reynolds numbers: from the bottom curve to the top one, $\operatorname{Re}=190,200,207,213,219$, and 225, respectively.

peak occurs increases linearly with respect to Re. Recalling that the transient energy growth in the considered case is due to the $\mathrm{KH}$ amplification along the separation streamline, one can assume that the increase in $G(t)$ is due to the increase in the size of the bubble with the Reynolds number. Indeed, plotting the bubble size versus the Reynolds number, a linear dependence is recovered, as shown in Fig. 26.

In the slightly unstable case $(\mathrm{Re}=225)$, a linear energy gain equal about to $10^{12}$ has been found, as shown by the top curve in Fig. 25, which is a very high amplification with respect to the data available in the literature for other flow configurations. For instance, in Ref. 8, for a separated flow over a bump the authors found a peak value about equal to $10^{9}$ for the critical Reynolds $\operatorname{Re}_{\delta}=590$, whereas here a growth of order of magnitude $10^{12}$ is observed for $\operatorname{Re}_{\delta}=225$. Such a difference is clearly dependent on the bubble size and could also be due to the absence of solid boundaries bordering the bubble, which allows the perturbation to amplify itself along the separation streamline from the separation point to the reattachment one.

In order to further investigate the linear unstable dynamics of an initial perturbation, the evolution of the optimal perturbation at $\mathrm{Re}=225$ has been studied. As shown in Fig. 27 , the perturbation is initially convected downstream by the mean flow as a localized wave packet. However, unlike the stable case previously analyzed, a second wave packet is generated due to the amplification of the disturbances carried back by the recirculation bubble. Such a disturbance shedding cycle is not due to an absolute instability of the velocity profiles within the bubble, as assessed by a local eigenvalue analysis, but to the global characteristics of the flow. It is

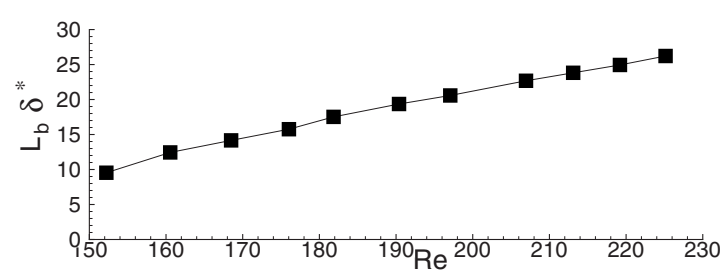

FIG. 26. Dimensional bubble size $L_{b} \delta^{*}$ vs Re.
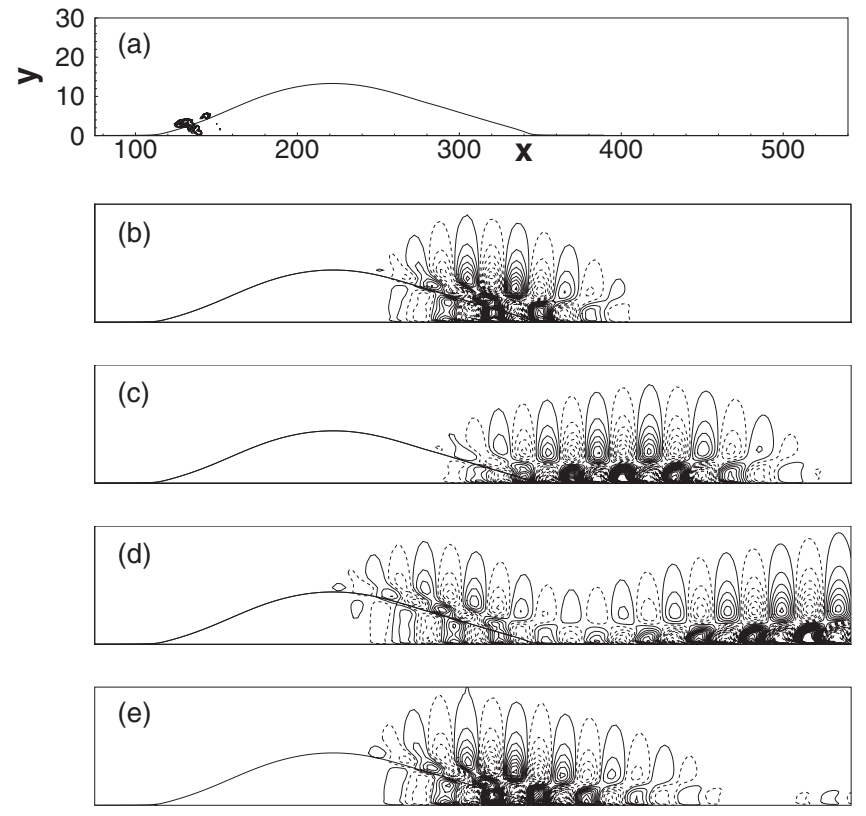

FIG. 27. Snapshots of the streamwise perturbation velocity contours for the optimal perturbation obtained by the global eigenvalue analysis for $\mathrm{Re}$ $=225$ at (a) $t=0$, (b) $t=450$, (c) $t=650$, (d) $t=850$, and (e) $t=1050$. Solidline contours indicate positive velocities; dashed-line ones are associated with negative velocities.

worth to notice that the subcritical transient growth mechanism in a nonlinear framework analyzed in Sec. IV A 3 seems to be not far from the asymptotically unstable mechanism shown here. In the subcritical case, the nonlinear terms being able to transfer energy among different modes, a wave packet cycle begins to be established until it decays due to the asymptotical damping of the perturbation. Thus, the generation of wave packets by the cyclic transfer of energy from the upstream part to the downstream part of the bubble and vice versa seems to be a feature of the stability dynamics of such a separated flow.

Focusing now on the asymptotic behavior of the flow at different Reynolds numbers, one can observe in Fig. 25 that each energy gain curve is affected by a modulation. Such a modulation, also named beating or flapping frequency, is a well-known feature of separated flows, ${ }^{8,28}$ although it has been found for the first time in a falling curtain flow. ${ }^{18}$ Such a phenomenon is due to the interaction of the most unstable modes of the spectrum. In Fig. $4(\mathrm{Re}=200)$, one can observe two modes, labeled $\omega_{1}$ and $\omega_{2}$, having comparable amplification rate. Since such modes are associated with similar eigenvectors, they are able to interact resulting in the low frequency modulation observed for the energy gain curve at $\mathrm{Re}=200$. In fact, since the real parts of these eigenmodes differ at about $\delta \omega_{r}=0.006$, their interaction results in a wave packet of period $T=2 \pi / \delta \omega_{r} \approx 1000$, which corresponds to the modulation shown in Fig. 28 by the energy gain curve for $\operatorname{Re}=200$ (dashed line). Moreover, it appears from Fig. 25 that two frequencies can be identified for each energy gain curve with $R e \geq 213$. For instance, at $R e=225$, the first frequency (labeled as I) corresponds to the low-frequency beating found in the previous case, having a period of about $T \approx 850$ (see the solid line in Fig. 28). The second frequency 


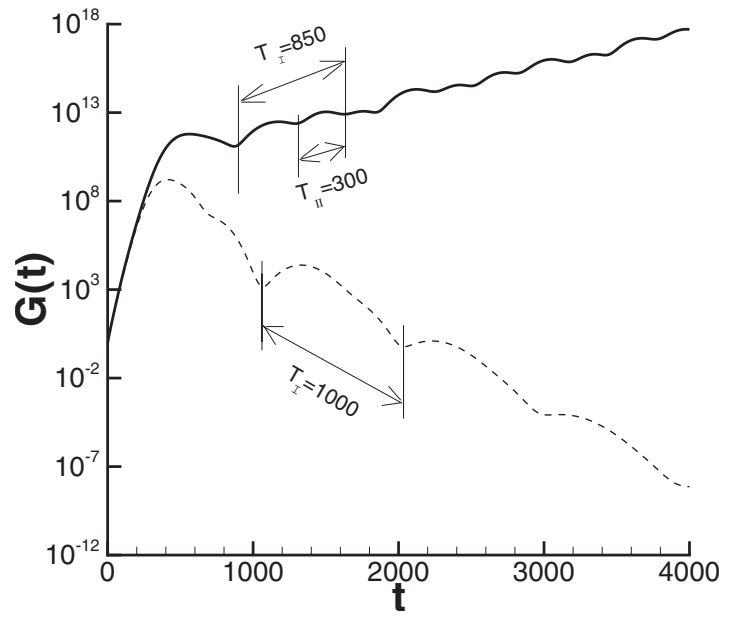

FIG. 28. Optimal energy gain curves obtained by the global eigenvalue analysis with $N=600$ modes for $\operatorname{Re}=225$ (solid line) and $\operatorname{Re}=200$ (dashed line).

(labeled as II) is slightly higher than the first one, having a period of about $T \approx 300$. Indeed, by looking at the spectrum at $\mathrm{Re}=225$, provided in Fig. 24, one can notice that the three most unstable modes have very similar amplification rate, leading to cancellation as in the stable case. Since their real parts differ at about $\delta \omega_{r_{\mathrm{I}}}=\omega_{r_{3}}-\omega_{r_{1}} \approx 0.0075$ and $\delta \omega_{r_{\mathrm{II}}}=\omega_{r_{2}}-\omega_{r_{3}} \approx 0.02$, they result, respectively, in two wave packets of period $T_{\mathrm{I}}=2 \pi / \delta \omega_{r_{\mathrm{I}}} \approx 850$ and $T_{\mathrm{II}}=2 \pi / \delta \omega_{r_{\mathrm{II}}} \approx 300$. It is worth to notice that due to the linearity of the global eigenvalue analysis, for large values of $t$ the beating induced by the two most unstable modes (frequency II) dominates the other one, as reported in Fig. 28. The sensitivity of such results with respect to the grid resolution and the domain length is discussed in the Appendix.

\section{Nonlinear dynamics}

The physical mechanism governing the beating phenomenon has been studied by performing several numerical simulations with increasing Reynolds numbers. DNS predicts transition at $\mathrm{Re}=230$, which is close but not exactly equal to the critical Reynolds number obtained by the linear analysis, namely, $R e=225$. Such a discrepancy might be due to a stabilizing effect of the convective outflow boundary conditions, as conjectured in Ref. 8, or to an imperfect convergence of the convective branch of the eigenvalue spectrum, which is very sensitive to numerical parameters, or to the different numerical discretizations used in the global eigenvalue analysis and DNS. Superposing the optimal perturbation obtained by the transient growth analysis upon the base flow at $R e=225$, the time evolution of the energy gain has been computed by DNS. In Fig. 29 it is possible to observe that two modulations affect the energy gain curve after the transient has passed, with periods $T_{\mathrm{I}} \approx 1200$ and $T_{\text {II }} \approx 350$. Such results are in good agreement with the ones found by using the global eigenvalue analysis.

It is worth to notice that the linear peak value of the energy gain $10^{12}$ (see Fig. 28) is not reached in the nonlinear case shown in Fig. 29, the predicted optimal growth being so high that the energy saturates before. In the considered case,

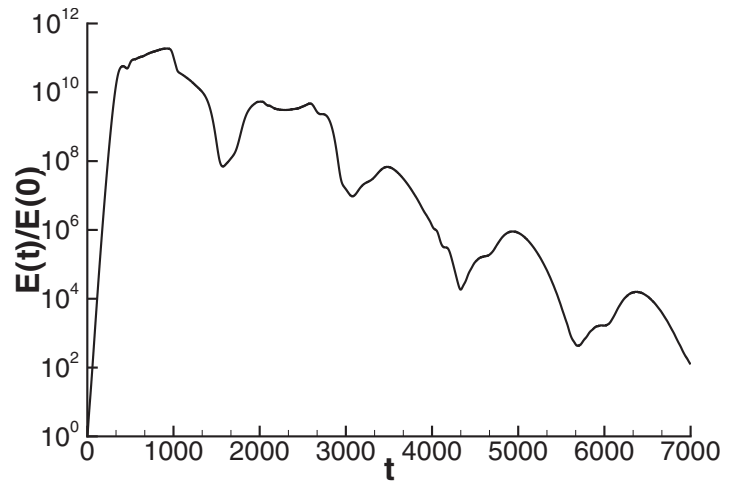

FIG. 29. Time evolution of the energy gain of an initial optimal perturbation of order of magnitude $A_{0}=10^{-6}$ computed by the DNS at $\operatorname{Re}=225$.

by injecting an initial perturbation with order of magnitude $10^{-6}$, which corresponds to an initial energy of order of magnitude $10^{-12}$, such a perturbation amplifies itself up to a factor of $10^{11}$, reaching amplitudes about equal to $10^{-1}$, so that the nonlinear effects are not negligible. However, as the amplitudes of the perturbations begin to decrease due to asymptotic stability, a linear dynamics is eventually recovered.

At supercritical Reynolds numbers, saturation occurs earlier due to the interactions of exponentially growing wave packets and it is maintained asymptotically. In order to assess if the two flapping frequencies are a feature of the considered flow also at supercritical conditions, the power spectrum in time of the evolving perturbation $u^{\prime}$ at a point within the bubble $(x=339, y=1.4)$ has been computed for $\mathrm{Re}=230$. Figure 30 shows the power density spectrum obtained by the Welch periodogram/fast Fourier transform method of $u^{\prime}$ on a sampling period of $T=40000$ divided in eight partially overlapped windows. A Hamming window function has been used on each data segment. Two frequency ranges where the energy is mostly located are found. The higher frequency range corresponds to TS waves, since the pulsations in the range $0.06<\omega_{r}<0.12$ correspond to the globally unstable modes of Fig. 24. Moreover, in the low-frequency region it is possible to recover three leading pulsations which are close to the flapping frequencies I and II and to their difference, respectively $\left(\omega_{\mathrm{I}} \approx 0.005, \quad \omega_{\mathrm{II}} \approx 0.014, \quad\right.$ and $\omega_{\mathrm{III}}=\omega_{\mathrm{II}}-\omega_{\mathrm{I}}$ $\approx 0.009$ ).

In order to shed light on the onset of the beating phenomenon, the dependence of such modulations on the Reynolds number has been investigated. The primary flapping

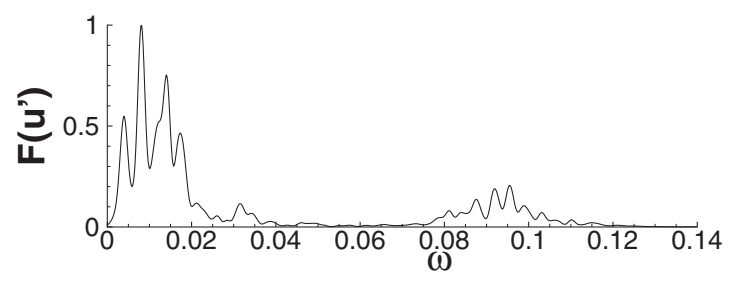

FIG. 30. Power density spectrum in time of the streamwise perturbation velocity, $u^{\prime}$, obtained by a Welch method on a sampling period of $T$ $=40000$ divided in eight partially overlapped windows, for $\mathrm{Re}=230$ at $x=339, y=1.4$. 


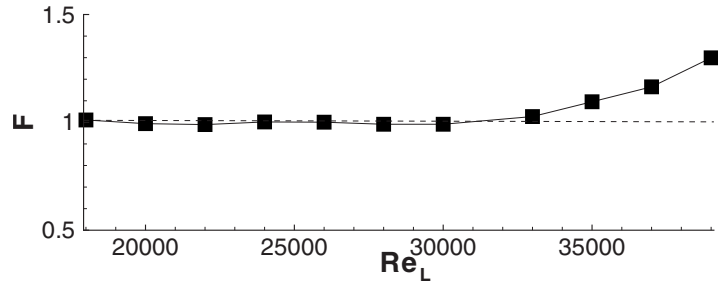

FIG. 31. Values of the dimensionless flapping frequencies, $F$, vs $\operatorname{Re}_{L}$. The values of $\operatorname{Re}$ at the black square points are 152, 160, 168, 176, 182, 191, $200,207,213,220$, and 225 , respectively.

frequencies have been computed by means of the global eigenvalue analysis at several Reynolds numbers lying in the range of $150 \leq \operatorname{Re} \leq 225$. In order to eliminate the dependence of the frequencies on $\delta^{*}$, a Reynolds number based on the reference length $L=0.05 \mathrm{~m}, \mathrm{Re}_{L}=U_{\infty} L / \nu$ has been employed, the corresponding dimensionless frequency being $F=L / \delta^{*} f$. Plotting the frequency values versus $\mathrm{Re}_{L}$, as shown in Fig. 31, it appears that $F \approx 1$ for $\operatorname{Re}_{L}$ $\leq 33000(\operatorname{Re} \leq 207)$, whereas for $\operatorname{Re}_{L} \geq 35000(\operatorname{Re} \geq 213)$, which is the threshold for the onset of the secondary flapping frequency, the frequency $F$ slightly increases. A possible explanation of such a behavior is here provided, which concerns the physical mechanism governing the onset of the flapping frequencies in separated flows over a flat plate. Recalling that the beating is due to the interaction of two modes presenting similar eigenvectors, and that such an interaction has been observed in several separated flows, such as cavityinduced and bump-induced separations, ${ }^{8,28}$ it can be argued that a separated region, which is able to carry back the perturbation, could play an active role in the generation of the interaction between modes. In fact, a part of the perturbation located in the separated region could be convected upstream within the bubble, where it is likely to interact with the main part of the wave packet, which is being convected downstream along the separation streamline. Therefore, one may assume that the beating frequency would be proportional to $1 / t_{L}$, where $t_{L}$ is the time needed by the mean flow to carry back such a wave packet from the reattachment point to the separation streamline,

$$
F \approx 1 / t_{L} \approx U_{b} / L_{b},
$$

where $L_{b}$ is the size of the bubble and $U_{b}$ the velocity of the base flow within the bubble. By measuring the variation in the bubble size and in the velocity at the first grid point from the wall where the maximum backflow is recovered at dif-

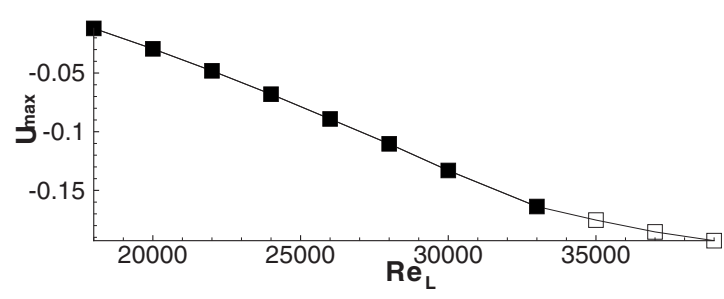

FIG. 32. Values of the maximum backflow velocity within the bubble vs $\mathrm{Re}_{L}$. The values of $\mathrm{Re}$ at the black square points are 152, 160, 168, 176, 182, 191, 200, and 207, respectively, whereas at the empty square points $\mathrm{Re}=213,220$, and 225, respectively.
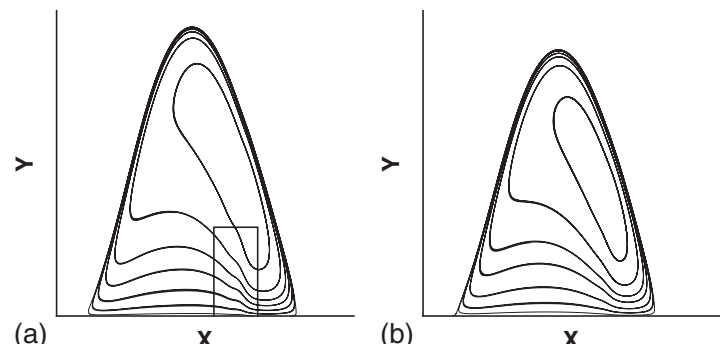

FIG. 33. Streamlines within the separated region for two base flows at (a) $\operatorname{Re}=213$ and (b) $R e=207$. Within the panel, the inflections of the streamlines for $\operatorname{Re}=213$ are shown.

ferent Reynolds numbers, it appears that both $L_{b}$ and $U_{b}$ vary linearly with respect to $\operatorname{Re}_{L}$ when $\operatorname{Re}_{L}<35000(\operatorname{Re}<213$, see Figs. 26 and 32, respectively). Such a linear dependence is broken when $\operatorname{Re}_{L} \geq 35000$ due to some topological changes in the base flow. Thus, as long as $\operatorname{Re}_{L}<35000$, the ratio between two frequencies $F_{1}$ and $F_{2}$ at two Reynolds numbers $\operatorname{Re}_{L_{1}}$ and $\operatorname{Re}_{L_{2}}$ could be written as

$$
\frac{F_{2}}{F_{1}} \approx \frac{t_{L_{1}}}{t_{L_{2}}} \approx \frac{U_{b_{2}}}{U_{b_{1}}} \frac{L_{b_{1}}}{L_{b_{2}}}=\frac{\operatorname{Re}_{L_{2}}}{\operatorname{Re}_{L_{1}}} \frac{\operatorname{Re}_{L_{1}}}{\operatorname{Re}_{L_{2}}}=1,
$$

meaning that the flapping frequency, $F$, remains constant when the Reynolds number varies, confirming the results shown in Fig. 31 for $\operatorname{Re}_{L}<35000$.

Concerning the dynamics at $\operatorname{Re}_{L}=35000 \quad(\operatorname{Re}=213)$, looking at the corresponding base flow in Fig. 33(a), one can see an inflection of the streamlines within the bubble, which is not observed for smaller values of the Reynolds number [see Fig. 33(b) corresponding to Re=207]. The DNS shows that when the flow is perturbed or when the Reynolds number is increased, such a topological change originates a small separation which splits the bubble in two smaller ones, labeled as part A and part B in Fig. 34. It is possible to assume that the onset of the flapping frequency II at such a Reynolds number is a consequence of the splitting of the bubble in two smaller ones, which could carry back the perturbations at two different rates generating two distinct modulations of the energy gain curve. Indeed, one can notice that the ratio of the

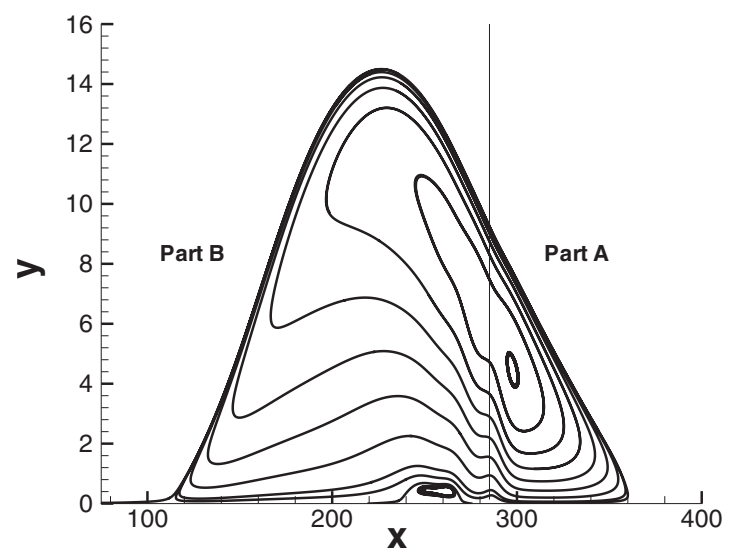

FIG. 34. Streamlines within the separated region for the base flow at $\operatorname{Re}=230$. The presence of a secondary bubble within the first one divides the bubble in two parts, labeled A and B. 


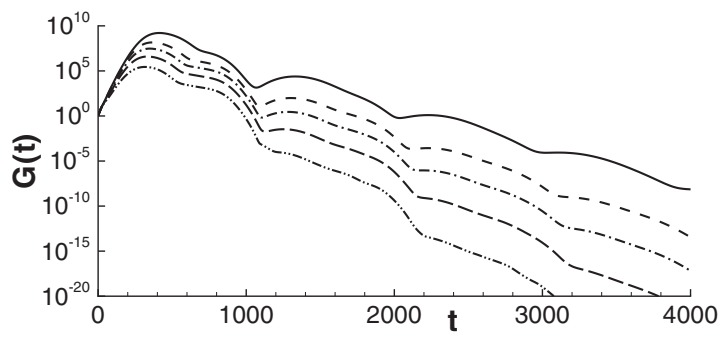

FIG. 35. Energy gain curves computed by the global eigenvalue analysis for $\mathrm{BF} 1, \mathrm{BF} 2, \mathrm{BF} 3, \mathrm{BF} 4$, and BF5 (from top to bottom).

size of bubble A with respect to the size of the bubble B $\left(L_{\mathrm{B}} / L_{\mathrm{A}} \approx 2.5\right)$ is very close to the ratio of the two flapping frequencies $\left(\omega_{\mathrm{II}} / \omega_{\mathrm{I}} \approx 2.8\right)$, supporting such an assumption. Moreover, the bubble splitting would also explain why a higher value of the flapping frequency $\mathrm{I}$ is recovered for $\mathrm{Re}_{L} \geq 35$ 000. Indeed, assuming that the beating I is generated by the part B of the bubble (while the beating II is generated by the part A), one can argue that such a bubble, which is smaller than the original one, would be able to carry back disturbances in a smaller time, originating a higher primary beating frequency.

In order to validate such a model for the case of smaller bubbles, the onset of the flapping frequency has been investigated for $\mathrm{BF} 2, \mathrm{BF} 3, \mathrm{BF} 4$, and BF5. Figure 35 provides the energy gain curves computed by the global eigenvalue analysis for BF1, BF2, BF3, BF4, and BF5 (from top to bottom), showing that a low-frequency modulation is recovered for all of the considered separated flows. The dependence of the flapping frequency, $F$, on the ratio $U_{b} / L_{b}$ has been investigated, where $U_{b}$, representing the upstream convection velocity of the base flow, has been measured within the bubble at the first grid point from the wall where the maximum backflow is recovered. Figure 36 shows that $F$ has approximatively a linear variation versus the ratio $U_{b} / L_{b}$, validating the conjecture of Eq. (15). Finally, it should be noticed that the onset of the flapping modulation in the energy gain curve for BF5 (see the bottom curve in Fig. 35) is associated with very low levels of the normalized energy, about equal to $10^{-5}$. Therefore, it is likely that the beating could not be observed in an experimental framework for small size bubbles. Moreover, it has been verified that for a smaller bubble, having an aspect ratio of about $h / L \approx 0.01$, the flapping phenomenon is inhibited probably due to the very narrow shape of the separated zone. For larger bubbles, the flapping phenomenon is associated with higher levels of the

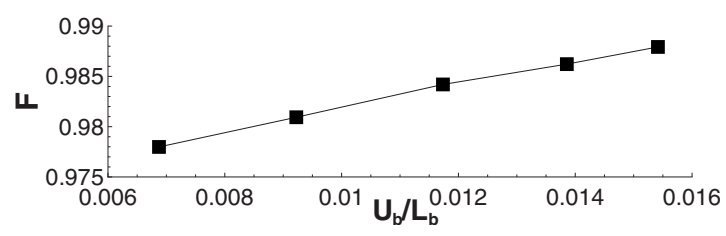

FIG. 36. Value of the flapping frequency $F$ vs the ratio $U_{b} / L_{b}$ computed by the global eigenvalue analysis for $\mathrm{BF} 1, \mathrm{BF} 2, \mathrm{BF} 3, \mathrm{BF} 4$, and $\mathrm{BF} 5$ (from right to left).

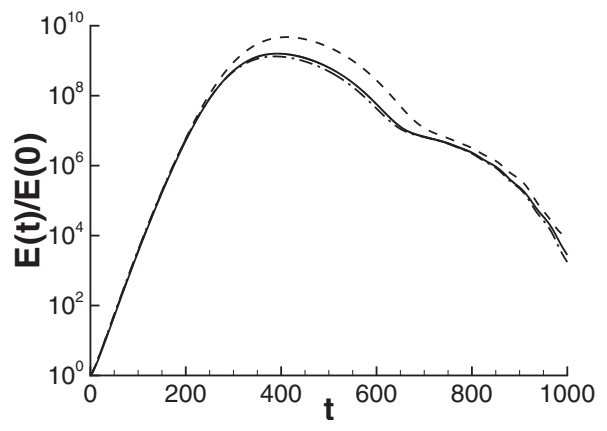

FIG. 37. Energy gain curves obtained by the DNS with a $(251 \times 75)$ grid (dashed line), a $(501 \times 150)$ grid (solid line), and a $(1001 \times 300)$ grid (dashed-dotted line).

energy, in some cases affecting the dynamics of the separated flow also in the presence of nonlinear effects, and becoming observable in experimental measurements. ${ }^{36}$

\section{CONCLUSIONS}

The transient and asymptotical dynamics of a large separation bubble over a flat plate has been studied in order to investigate the role of non-normality and nonlinearity of the differential operator in the stability of such a flow. Two numerical tools have been employed, namely, the global eigenvalue analysis and the DNS; moreover, a continuation procedure based on Newton's iteration has been used to compute the steady-state solutions of the NS equations at slightly supercritical Reynolds numbers. Linear eigenvalue analysis, as well as numerical simulations with weakly nonlinear perturbations, have shown that the non-normality of convective modes of the NS operator allows the bubble to act as a strong amplifier of small disturbances. In particular, the initial perturbation allowing the largest energy gain is found to be placed close to the separation point and to be amplified by a Kelvin-Helmholtz mechanism while being convected downstream. Indeed, the front part of the bubble shows a high sensitivity to external noise, as observed in simulations with white noise disturbances superposed upon the whole base flow or upon the inflow Blasius profile. For finite amplitude initial perturbations, the strong linear transient amplification saturates due to nonlinear interactions between modes. Furthermore, such an energy exchange between modes induces the bubble to establish a wave packet cycle, characterized by the shedding of two wave packets of comparable amplitude. Such a cycle is similar to the one occurring at supercritical Reynolds numbers, but it is eventually damped when the

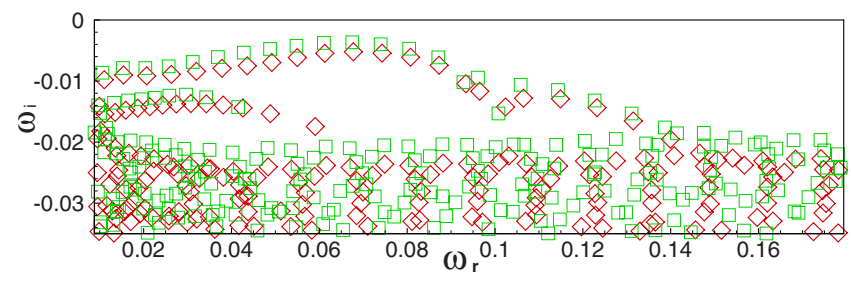

FIG. 38. (Color online) Global spectrum obtained for $\mathrm{Re}=200$ with a $(200 \times 40)$ grid (squares) and a $(250 \times 48)$ grid (diamonds) . 


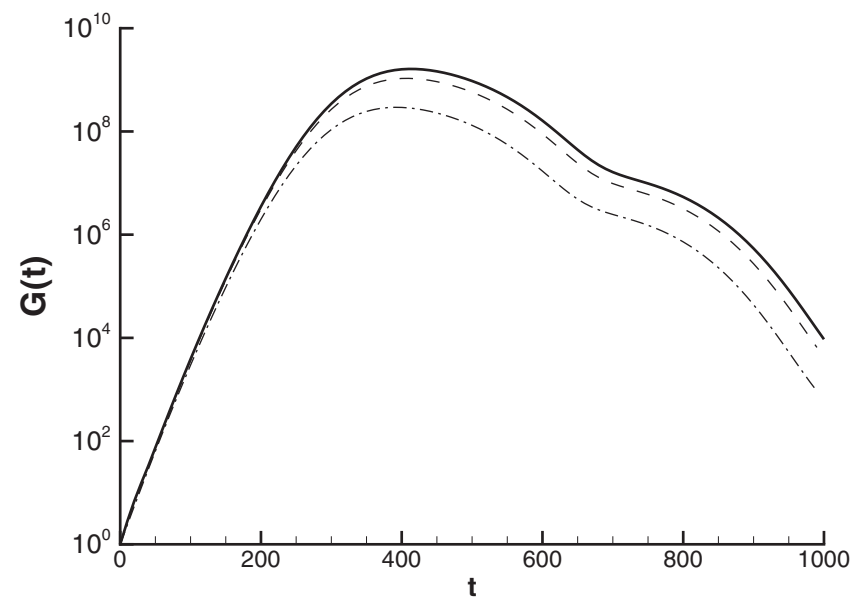

FIG. 39. Optimal energy gain curve at $\mathrm{Re}=200$ computed with $N=600$ modes (solid line, reference case), $N=500$ modes (dashed line), and $N=300$ modes (dashed-dotted line).

flow is asymptotically stable. Nonlinear interactions contribute also to the excitation of a convectively unstable mode in the attached-flow region due to the high sensitivity of the boundary layer, inducing a further transient amplification of finite amplitude perturbations as well as an asymptotical instability at slightly subcritical Reynolds numbers.

Concerning the unstable dynamics of the considered flow, topological flow changes on the base flow have been found to occur close to transition, supporting the hypothesis of some authors $^{12,35}$ that the unsteadiness of separated flows could be due to structural changes within the bubble. Furthermore, non-normality effects have shown to play an active role at large times. In fact, due to the superposition of two convective non-normal modes, a low-frequency oscillation known as flapping frequency appears. Close to transition, when topological changes occur in the flow, a secondary flapping frequency appears as well. A possible explanation of such a behavior has been provided, in which it is assumed that the oscillations are due to the interaction of the main wave packet with the perturbations carried upstream by the backflow inside the bubble. A scaling law based on the previous assumption is able to predict accurately the dependence of the flapping frequency on the Reynolds number and the onset of the secondary frequency close to transition. Future works will focus on the physical description of the flapping phenomenon in order to provide a further validation of the above conjecture and to test it in a more realistic threedimensional case. Preliminary DNS results show that the

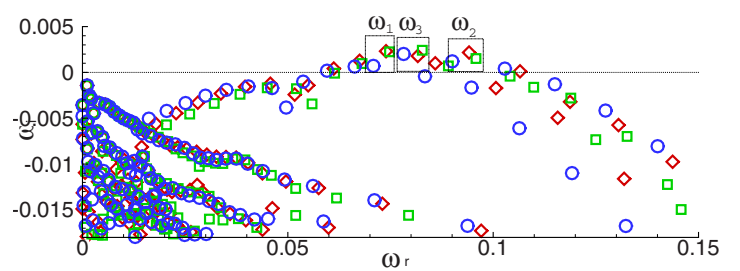

FIG. 40. (Color online) Spectra obtained at $\mathrm{Re}=225$ for three domain lengths: $L_{1}=430$ (diamonds), $L_{2}=480$ (squares), and $L_{3}=530$ (circles).

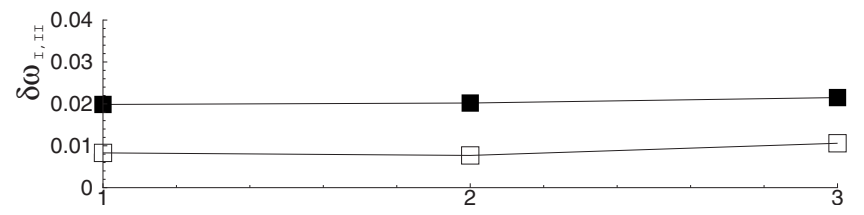

FIG. 41. Sensibility of the value of the flapping frequencies I (white squares) and II (black squares) to the grid resolution: $N_{x}=260, N_{y}=48$ (point 1); reference case, $N_{x}=270, N_{y}=50$ (point 2); and $N_{x}=300, N_{y}=56$ (point 3).

flapping frequencies are recovered also in a threedimensional configuration, and that their values are close to the ones here obtained for the 2D case.

\section{ACKNOWLEDGMENTS}

This work was performed using HPC resources from GENCI-CCRT/IDRIS (Grant 2009-022188). The authors would like to thank R. Verzicco for providing the secondorder accurate version of the DNS code, F. Alizard for providing the code for the global eigenvalue analysis, and $\mathrm{M}$. Napolitano and A. Lerat for making possible the cooperation between the two laboratories in which this work has been developed. This research has been supported by MIUR and Politecnico di Bari under contracts CofinLab2001 and PRIN2007.

\section{APPENDIX: CONVERGENCE STUDIES}

A grid convergence study for the DNS has been performed by computing the energy growth of the optimal perturbation for the separated flow at $\mathrm{Re}=200$ using three grids with $251 \times 75,501 \times 150$, and $1001 \times 300$ cells, respectively. As shown in Fig. 37, the results obtained using the second and the third grids are very close to each other, so that the $501 \times 150$ grid, which has been used in all of the computations, can be considered adequate to capture the energy growth of the disturbances accurately.

Concerning the global model, Fig. 38 shows the spectra obtained for the separated flow at $\mathrm{Re}=200$ using two grids with $200 \times 40$ and $250 \times 48$ collocation points, respectively (computations with a considerably finer grid are not possible due to memory requirements). The shape of the spectra computed with the two grids does not show remarkable differences, although the modes are found to slightly change their position. Nevertheless, it is well known that a pointwise convergence of the global spectra with respect to grid resolution for highly nonparallel flows cannot be reached due to the high non-normality of the operator, ${ }^{34}$ see, for instance, the

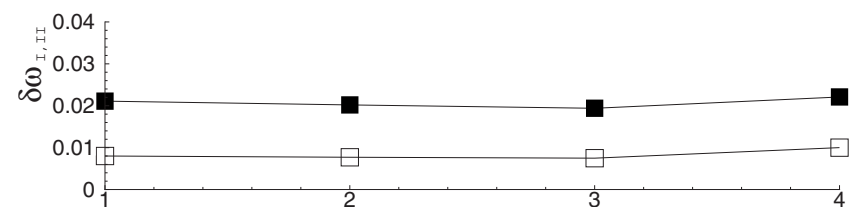

FIG. 42. Sensibility of the value of the flapping frequencies I (white squares) and II (black squares) to the domain length: $N_{x}=270, N_{y}=50$, $L_{x}=430$ (point 1); reference case, $N_{x}=270, N_{y}=50, L_{x}=480$ (point 2); $N_{x}=270, N_{y}=50, L_{x}=530$ (point 3); and $N_{x}=300, N_{y}=50, L_{x}=580$ (point 4). 
sensitivity study provided in Ref. 8 . Moreover, the sensitivity of the optimal energy gain, $G(t)$, with respect to the number of modes chosen for its evaluation [see Eq. (11)] has been investigated. Figure 39 shows the optimal energy gain curves obtained by choosing $N=300$ (dashed-dotted line), $N=500$ (dashed line), and $N=600$ modes among the $N_{t}$ total modes employed for the global eigenvalue analysis. The modes which are affected by oscillations have been discarded and the solution shows a clear tendency toward convergence.

Finally, the sensitivity of the computed flapping frequencies with respect to the grid resolution and the domain length has been studied. It is noteworthy that, although the spectra corresponding to different domain lengths do present some differences, as shown in Fig. 40, due to the different streamwise extension of the modes, the global model is able to accurately predict the overall stability features of the flow. In fact, the computations demonstrate that approximatively the same two distinct frequencies are recovered for different grid resolutions (see Fig. 41) and domain lengths (see Fig. 42 ), the influence of such parameters on $\delta \omega_{r_{\mathrm{I}}}$ and $\delta \omega_{r_{\mathrm{II}}}$ being negligible.

${ }^{1}$ P. Schmid and D. Henningson, Stability and Transition in Shear Flows (Springer, New York, 2001).

${ }^{2}$ P. J. Schmid, "Nonmodal stability theory," Annu. Rev. Fluid Mech. 39, 129 (2007).

${ }^{3} \mathrm{U}$. Rist and U. Maucher, "Direct numerical simulation of 2-d and 3-d instability waves in a laminar separation bubble," AGARD-CP. 551, 361 (1994).

${ }^{4}$ D. Barkley, G. M. Gomes, and R. D. Henderson, "Three-dimensional instability in flow over a backward-facing step," J. Fluid Mech. 473, 167 (2002).

${ }^{5}$ J.-F. Beaudoin, O. Cadot, J.-L. Aider, and J. E. Wesfreid, "Threedimensional stationary flow over a backward-facing step," Eur. J. Mech. B/Fluids 23, 147 (2004).

${ }^{6} \mathrm{H}$. Blackburn, D. Barkley, and S. Sherwin, "Convective instability and transient growth in flow over a backward-facing step," J. Fluid Mech. 603, 271 (2008).

${ }^{7}$ L. Marino and P. Luchini, "Adjoint analysis of the flow over a forwardfacing step," Theor. Comput. Fluid Dyn. 23, 37 (2009).

${ }^{8}$ U. Ehrenstein and F. Gallaire, "Global low-frequency oscillations in a separating boundary-layer flow," J. Fluid Mech. 614, 315 (2008).

${ }^{9}$ F. Gallaire, M. Marquillie, and U. Ehrenstein, "Three-dimensional transverse instabilities in detached boundary-layers," J. Fluid Mech. 571, 221 (2007).

${ }^{10} \mathrm{M}$. Marquillie and U. Ehrenstein, "On the onset of nonlinear oscillations in a separating boundary-layer flow," J. Fluid Mech. 490, 169 (2003).

${ }^{11}$ P. T. Williams and A. J. Baker, "Numerical simulations of laminar flow over a 3D backward-facing step," Int. J. Numer. Methods Fluids 24, 1159 (1997).

${ }^{12} \mathrm{~V}$. Theofilis, S. Hein, and U. Dallmann, "On the origins of unsteadiness and three dimensionality in a laminar separation bubble," Philos. Trans. R. Soc. London, Ser. A 358, 3229 (2000).

${ }^{13} \mathrm{~N}$. Cherry, R. Hillier, and M. Latour, "Unsteady measurements in a separated and reattaching flow," J. Fluid Mech. 144, 13 (1984).

${ }^{14}$ L. Pauley, P. Moin, and W. Reynolds, "The structure of two-dimensional separation," J. Fluid Mech. 220, 397 (1990).
${ }^{15}$ F. Alizard, S. Cherubini, and J. C. Robinet, "Sensitivity and optimal forcing response in separated boundary layer flows," Phys. Fluids 21, 064108 (2009).

${ }^{16}$ O. Marquet, D. Sipp, J.-M. Chomaz, and L. Jacquin, "Amplifier and resonator dynamics of a low-Reynolds-number recirculation bubble in a global framework," J. Fluid Mech. 605, 429 (2008)

${ }^{17}$ A. V. Dovgal, V. V. Kozlov, and A. Michalke, "Laminar boundary layer separation: Instability and associated phenomena," Prog. Aerosp. Sci. 30, 61 (1994).

${ }^{18}$ P. J. Schmid and D. S. Henningson, "On the stability of a falling liquid curtain,” J. Fluid Mech. 463, 163 (2002).

${ }^{19}$ E. Akervik, U. Ehrenstein, F. Gallaire, and D. S. Henningson, "Global two-dimensional stability measures of the flat plate boundary-layer flow," Eur. J. Mech. B/Fluids 27, 501 (2008).

${ }^{20}$ F. Alizard and J.-C. Robinet, "Spatially convective global modes in a boundary layer," Phys. Fluids 19, 114105 (2007).

${ }^{21} \mathrm{U}$. Ehrenstein and F. Gallaire, "On two dimensional temporal modes in spatially evolving open flows: The flat-plate boundary layer," J. Fluid Mech. 536, 209 (2005).

${ }^{22} \mathrm{R}$. Verzicco and P. Orlandi, "A finite-difference scheme for the threedimensional incompressible flows in cylindrical coordinates," J. Comput. Phys. 123, 402 (1996).

${ }^{23} \mathrm{P}$. Chu and C. Fan, "A three point sixth-order non uniform combined compact difference scheme," J. Comput. Phys. 148, 663 (1999).

${ }^{24}$ L. S. Tuckerman and D. Barkley, "Bifurcation analysis for timesteppers," Numerical Methods for Bifurcation Problems and Large-Scale Dynamical Systems (Springer, New York, 2000), Vol. 119, pp. 543-466.

${ }^{25} \mathrm{Y}$. Na and P. Moin, "The structure of wall-pressure fluctuations in turbulent boundary layers with adverse pressure gradient and separation," J. Fluid Mech. 377, 347 (1998).

${ }^{26}$ E. Åkervik, L. Brandt, D. S. Henningson, J. Hoepffner, O. Marxen, and P. Schlatter, "Steady solutions of the Navier-Stokes equations by selective frequency damping," Phys. Fluids 18, 068102 (2006).

${ }^{27}$ R. Lehoucq, D. Sorensen, and C. Yang, ARPACK Users' Guide: Solution of Large Scale Eigenvalue Problems with Implicitly Restarted Arnoldi Methods (SIAM, Philadelphia, 1997).

${ }^{28}$ E. Åkervik, J. Hoepffner, U. Ehrenstein, and D. S. Henningson, "Optimal growth, model reduction and control in a separated boundary-layer flow using global eigenmodes," J. Fluid Mech. 579, 305 (2007).

${ }^{29}$ C. P. Häggmark, A. A. Bakchinov, and P. H. Alfreddson, "Experiments on a two-dimensional laminar separation bubble," Philos. Trans. R. Soc. London, Ser. A 358, 3193 (2000).

${ }^{30} \mathrm{~J}$. H. Watmuff, "Evolution of a wave packet into vortex loops in a laminar separation bubble," J. Fluid Mech. 397, 119 (1999).

${ }^{31}$ O. Marxen, M. Lang, U. Rist, and S. Wagner, "A combined experimental/ numerical study of unsteady phenomena in a laminar separation bubble," Flow, Turbul. Combust. 71, 133 (2003).

${ }^{32} \mathrm{C}$. Cossu and J. Chomaz, "Global measure of local convective instabilities," Phys. Rev. Lett. 78, 4387 (1997).

${ }^{33}$ L. Kaiktsis, G. E. Karniadakis, and S. A. Orszag, "Unsteadiness and convective instabilities in two-dimensional flow over a backward-facing step," J. Fluid Mech. 321, 157 (1996).

${ }^{34}$ L. N. Trefethen and M. Embree, Spectra and Pseudospectra: The Behaviour of Nonnormal Matrices and Operators (Princeton University Press, Princeton, NJ, 2005).

${ }^{35}$ U. Dallmann, T. Herberg, H. Gebing, W.-H. Su, and H.-Q. Zhang, "Flow field diagnostics-Topological flow changes and spatio-temporal flow structure," AIAA Paper No. 95-0791, 1995.

${ }^{36}$ A. Dovgal and A. Sorokin, "Wave packets of controlled velocity perturbations at laminar flow separation," Proceedings of the VII IUTAM Symposium on Laminar-Turbulent Transition, IUTAM Bookseries (Springer, New York, 2009), Vol. 18. 